TRANSACTIONS OF THE

AMERICAN MATHEMATICAL SOCIETY

Volume 359, Number 11, November 2007, Pages 5207-5238

S 0002-9947(07)04204-3

Article electronically published on June 4, 2007

\title{
IWASAWA THEORY FOR $K(1)$-LOCAL SPECTRA
}

\author{
REBEKAH HAHN AND STEPHEN MITCHELL
}

\begin{abstract}
The Iwasawa algebra $\Lambda$ is a power series ring in one variable over the $p$-adic integers. It has long been studied by number theorists in the context of $\mathbb{Z}_{p}$-extensions of number fields. It also arises, however, as a ring of operations in $p$-adic topological $K$-theory. In this paper we study $K(1)$-local stable homotopy theory using the structure theory of modules over the Iwasawa algebra. In particular, for $p$ odd we classify $K(1)$-local spectra up to pseudo-equivalence (the analogue of pseudo-isomorphism for $\lambda$-modules) and give an Iwasawa-theoretic classification of the thick subcategories of the weakly dualizable spectra.
\end{abstract}

\section{INTRODUCTION}

Fix a prime $p$ and let $K$ denote the $p$-completed complex $K$-theory spectrum. The ring of operations $K^{0} K$ can be identified with a pro-group ring $\mathbb{Z}_{p}\left[\left[\Gamma^{\prime}\right]\right]$. Here $\Gamma^{\prime}$ is the group of $p$-adic Adams operations, isomorphic to $\mathbb{Z}_{p}^{\times}$. It splits uniquely as a product $\Gamma \times \Delta$, where $\Gamma$ corresponds to the units congruent to $1 \bmod p$, and $\Delta$ to the $(p-1)$-st roots of unity (if $p$ is odd) or $\{ \pm 1\}$ (if $p=2$ ). Furthermore, $\mathbb{Z}_{p}[[\Gamma]]$ is isomorphic to the power series ring $\Lambda=\mathbb{Z}_{p}[[T]]$ : the Iwasawa algebra. Then for any spectrum $X$ and $n \in \mathbb{Z}, K^{n} X$ is a compact module over the compact topological $\operatorname{ring} \Lambda[\Delta]$.

The Iwasawa algebra has long been studied by number theorists in connection with $\mathbb{Z}_{p}$-extensions of number fields. In particular, there is a beautiful classification theorem for finitely-generated $\Lambda$-modules that has been exploited to great advantage. It is exactly like the classical theorem for modules over a principal ideal domain, except that the classification only holds up to pseudo-isomorphism - the morphisms with finite kernel and cokernel.

In view of the isomorphism $K^{0} K \cong \Lambda^{\prime}$, the structure theory of $\Lambda$-modules can be applied to $K$-theory. In fact this observation is not new; it was pointed out thirty years ago by Bousfield [2] and Ravenel [18]. Somewhat surprisingly, however, the idea seems not to have been pursued further. The purpose of this paper is to consider $K$-theory systematically in terms of Iwasawa modules.

We work throughout in the category $L_{K(1)} \mathcal{S}$ of $K(1)$-local spectra. Loosely speaking, this category is obtained by formally inverting all morphisms of spectra that induce an isomorphism on $K^{*}$. Since $K^{*}$ is periodic, we need only consider $K^{\cdot}=K^{0} \oplus K^{1}$. We call a morphism $f: X \longrightarrow Y$ in $L_{K(1)} \mathcal{S}$ a pseudo-equivalence if its

Received by the editors June 14, 2005.

2000 Mathematics Subject Classification. Primary 55N15, 19L20.

Key words and phrases. K-theory, homotopy theory, Iwasawa algebra.

The second author was supported by a grant from the National Science Foundation.

(C)2007 American Mathematical Society 
cofibre $C_{f}$ is small, in the sense that the functor $\left[C_{f},-\right]$ takes arbitrary coproducts to direct sums. This is equivalent to requiring that $K^{\cdot} C_{f}$ is finite, and hence $f$ is a pseudo-equivalence if and only if $K f$ is a pseudo-isomorphism. However, the definition as stated is conceptually illuminating, and suggests a number of interesting generalizations.

In order to apply the classical theory of Iwasawa modules, we must first restrict ourselves to the thick subcategory $L_{K(1)}^{f i n} \mathcal{S}$ whose objects $X$ have $K \cdot X$ finitelygenerated over $\Lambda$. The objects of this category can be characterized in several interesting ways: as the spectra whose homotopy groups are finitely-generated over $\mathbb{Z}_{p}$; as the spectra $X$ such that the natural topology on $[-, X]$ is compact; and as the objects that are weakly dualizable, i.e., the double duality map $X \longrightarrow D^{2} X$ is an equivalence. In this last characterization the functional dual $D X=\mathcal{F}\left(X, S^{0}\right)$ can be replaced by the Brown-Comenetz dual, a spectrum-level analogue of the Pontrjagin dual. All this is explained in detail in $\S 8$.

We then obtain (1) a classification of objects in $L_{K(1)} \mathcal{S}$ up to pseudo-equivalence, and (2) a classification of the thick subcategories of $L_{K(1)}^{f i n} \mathcal{S}$ in Iwasawa-theoretic terms. For these results we assume $p$ is odd. Part of the difficulty at $p=2$ is that even the pure algebra of modules over $\Lambda[\Delta]$ is surprisingly complicated (see the discussion in [16]). We plan to return to the 2-primary case in a future paper.

The classification up to pseudo-equivalence in (1) is what one would expect (Theorem 9.3):

Theorem 1.1. Suppose $p$ is odd. For any $X \in L_{K(1)}^{f i n} \mathcal{S}$, there are pseudo-equivalences

$$
X \stackrel{\sim}{\longrightarrow} Y \stackrel{\sim}{\longleftarrow} Z
$$

with $Z$ an "elementary" spectrum; in other words, $K^{\cdot} Z$ is an elementary $\Lambda$-module (see $§ 2$ for the definition).

The thick subcategories are classified by the support of $K^{\prime} X$ in $S p e c \Lambda^{\prime}$. We call a subset of $\operatorname{Spec} \Lambda^{\prime}$ fit if it is closed under specialization and Tate twisting. Then (Theorem 10.2):

Theorem 1.2. Suppose $p$ is odd. The thick subcategories of $L_{K(1)}^{\text {fin }} \mathcal{S}$ are in bijective correspondence with the fit subsets of Spec $\Lambda^{\prime}$.

There are many interesting examples. For instance, one can start from a thick subcategory - say the spectra with finite homotopy groups, or the strongly dualizable objects - and then determine the corresponding fit subset. Conversely, one can start with a fit subset - say the fit subset generated by the irreducible distinguished polynomials, or by the semi-discrete primes - and then consider the corresponding thick subcategory.

We emphasize that $\S 4$ through $\S 8$ are based on the elegant work of HoveyPalmieri-Strickland on axiomatic stable homotopy theory 13, and of HoveyStrickland on $K(n)$-local spectra [12]. In fact, in most cases both the results and the proofs are specializations to $n=1$ of more general results from the latter memoir; our claims to originality in these sections are modest and few. Our justification for including this material here is threefold: first, we needed to translate everything into our Iwasawa-theoretic language. Second, the results of [12] often simplify considerably when $n=1$. We wanted to make our paper accessible without requiring the reader to digest all two hundred pages of [12], [13]. Therefore we have provided 
a mix of complete proofs and explicit references. Third, we include several results which, although probably known, are not treated explicitly in the literature.

Finally, we acknowledge that the term "Iwasawa theory" as used by number theorists entails far more than the mere algebra of $\Lambda$-modules considered here. It refers to the structure of certain specific modules arising in the study of number fields, elliptic curves and so on, and to the various deep relationships - some proved, some conjectural - between these modules and certain $p$-adic $L$-functions. However, the topology and the number theory are connected through algebraic $K$-theory, for example by taking $X \in L_{K(1)} \mathcal{S}$ to be the localization of the algebraic $K$-theory spectrum of a number ring. See 16.

Organization of the paper: $\S 2$ introduces the Iwasawa algebra, and the basic structure theorem for its modules. $\S 3$ introduces $p$-adic $K$-theory, and reviews some basic facts about $p$-completion and Ext-p-complete-abelian groups.

In $\S 4$ through $\S 8$ we study the $K(1)$-local homotopy category $L_{K(1)} \mathcal{S}$. As explained above, most of this material is translated directly from [12, [13] and also [10.

In $\S 9$ we introduce the notion of pseudo-equivalence in $L_{K(1)} \mathcal{S}$, and prove the classification theorem. In $\S 10$ we classify the thick subcategories of $L_{K(1)}^{f i n} \mathcal{S}$, with numerous examples. Note that in $\S 9$ and $\S 10, p$ is odd.

\section{The Iwasawa ALgeBra}

In this section we introduce the Iwasawa algebra $\Lambda$ and some basic properties of its modules, as well as the related algebra $\Lambda^{\prime}$. General references for this material include [17] and [21; see also the discussion in [16].

Let $\Gamma^{\prime}$ denote the automorphism group of $\mathbb{Z} / p^{\infty}$. Thus $\Gamma^{\prime}$ is canonically isomorphic to the $p$-adic units $\mathbb{Z}_{p}^{\times}$, with the isomorphism $c: \Gamma^{\prime} \longrightarrow \mathbb{Z}_{p}^{\times}$given by $\gamma(x)=c(\gamma) x$ for $\gamma \in \Gamma^{\prime}, x \in \mathbb{Z} / p^{\infty}$. In fact, if $A$ is any abelian group isomorphic to $\mathbb{Z} / p^{\infty}$, we again have a canonical isomorphism $\Gamma^{\prime} \cong A u t A$, defined in the same way. In particular, $\Gamma^{\prime}$ is canonically identified with the automorphism group of the group of $p$-power roots of unity in any algebraically closed field of characteristic different from $p$. Note also that there is a unique product decomposition $\Gamma^{\prime}=\Gamma \times \Delta$, where $\Gamma$ corresponds under $c$ to the units congruent to $1 \bmod p$ (resp. $1 \bmod 4$ ) if $p$ is odd (resp. $p=2$ ), and $\Delta$ corresponds to the $p-1$-st roots of unity (resp. \pm 1 ) if $p$ is odd (resp. $p=2$ ). The restriction of $c$ to $\Delta$ is denoted $\omega$ and called the Teichmuller character.

If $G$ is a profinite group, the associated pro-group ring $\mathbb{Z}_{p}[[G]]$ is the inverse limit of the ordinary group rings $\mathbb{Z}_{p}[G / U], U$ ranging over the open normal subgroups of $G$. We write $\Lambda$ for $\mathbb{Z}_{p}[[\Gamma]]$ and $\Lambda^{\prime}$ for $\mathbb{Z}_{p}\left[\left[\Gamma^{\prime}\right]\right]$. Let $\gamma_{0}$ be a topological generator of $\Gamma$. To be specific, we take $c\left(\gamma_{0}\right)=1+p$ if $p$ is odd, and $c\left(\gamma_{0}\right)=5$ if $p=2$. Then it was observed by Serre that there is an isomorphism of profinite rings

$$
\mathbb{Z}_{p}[[\Gamma]] \cong \mathbb{Z}_{p}[[T]]
$$

such that $\gamma_{0} \mapsto T+1$. Note that $\Lambda^{\prime}=\Lambda[\Delta]$.

The ring $\Lambda$ is a regular noetherian local domain of Krull dimension two. In particular, it has global dimension two, and every module over it admits a projective resolution of length at most two. It is also complete with respect to its maximal ideal $\mathfrak{m}=(p, T)$, with residue field $\mathbb{Z} / p$, and therefore is a profinite topological ring. 
The height one prime ideals are all principal, and are of two types. First there is the prime $p$, which plays a special role. Second, there are the prime ideals generated by the irreducible distinguished polynomials $f(T)$. Here a polynomial $f \in \mathbb{Z}_{p}[T]$ is distinguished if it is monic and $f(T)=T^{n} \bmod p, n=\operatorname{deg} f$. Note that each height one prime is now equipped with a canonical generator. We will usually not bother to distinguish between the ideal and its generator.

2.1. Modules over the Iwasawa algebra. An elementary cyclic module is a $\Lambda$ module that is either free of rank one or of the form $\Lambda / q^{i}$, where $q$ is an irreducible element; we can and will take $q$ to be either $p$ or an irreducible distinguished polynomial. A finitely-generated $\Lambda$-module $E$ is elementary if it is a direct sum of elementary cyclic modules. The primes $q$ and exponents $i$ that appear are uniquely determined by $E$, up to ordering.

A pseudo-isomorphism is a homomorphism of $\Lambda$-modules with finite kernel and cokernel. For example, suppose $f$ and $g$ are relatively prime elements of $\Lambda$. Then the natural homomorphism $\Lambda / f g \longrightarrow \Lambda / f \oplus \Lambda / g$ is a pseudo-isomorphism.

The main classification theorem reads:

Theorem 2.1. Let $M$ be a finitely-generated $\Lambda$-module. Then there is an elementary module $E$ and a pseudo-isomorphism $\phi: M \longrightarrow E$. Up to isomorphism, $E$ is uniquely determined by $M$.

Remark. If $M$ is a $\Lambda$-torsion module, then one can also find a pseudo-isomorphism $\psi: E \longrightarrow M$. In general, however, this is not true; if $M$ is $\Lambda$-torsionfree but not free, there is no pseudo-isomorphism from a free module to $M$.

Recall that the support of a module $M$, denoted $\operatorname{Supp} M$, is the set of primes $q$ such that $M_{q} \neq 0$. Note that $S u p p M$ is closed under specialization, i.e., if $q \in \operatorname{Supp} M$ and $q \subset q^{\prime}$, then $q^{\prime} \in \operatorname{Supp} M$. For example, the nonzero finite modules are the modules with support $\{\mathfrak{m}\}$, while the $\Lambda$-torsion modules are the modules with $(0) \notin$ Supp $M$.

Now if $E$ is the elementary module associated to $M$ as above, we have

$$
E=\Lambda^{r} \oplus \Lambda / q_{1}^{i_{1}} \oplus \ldots \oplus \Lambda / q_{s}^{i_{s}}
$$

where the $q_{i}$ 's are height one primes, not necessarily distinct. Thus $E$ is uniquely determined by its $\Lambda$-rank $r$ and its torsion invariants $\left(\left(q_{1}, i_{1}\right), \ldots,\left(q_{s}, i_{s}\right)\right)$. Note that the $q_{i}$ 's which occur, together with $\mathfrak{m}$, form the support of the $\Lambda$-torsion submodule $t M$. It will be convenient to use the following terminology:

(i) the frequency of a prime $q$ is the number of $q_{j}=q$;

(ii) the exponent of $q$ is the maximal $i_{j}$ occuring for $q_{j}=q$;

(iii) the multiplicity of $q$ is the sum of the $i_{j}$ 's for $q_{j}=q$.

We next summarize some properties of $\Lambda$-modules that will be used in the sequel. For further information, see [17. For example, the next proposition is 5.3.19 in [17].

Proposition 2.2. A $\Lambda$-module $M$ has projective dimension less than or equal to one if and only if $M$ has no finite $\Lambda$-submodules.

Proposition 2.3. If $M$ and $N$ are finitely-generated $\Lambda$-modules, then $\operatorname{Ext}_{\Lambda}^{2}(M, N)$ is finite.

Proof. Suppose first that $M$ is finite. Then $M$ is annihilated by some power of the maximal ideal $\mathfrak{m}$. Hence $\operatorname{Ext}_{\Lambda}^{k}(M, N)$ is finite for all $k$, since it is a finitelygenerated $\Lambda$-module annihilated by a power of $\mathfrak{m}$. In the general case it follows that 
the finiteness of $\operatorname{Ext}_{\Lambda}^{2}(M, N)$ is invariant under pseudo-isomorphisms of $M$, and we therefore reduce to the case when $M$ is an elementary module. Since elementary modules have projective dimension at most one, this completes the proof.

Proposition 2.4. Let $N$ be a submodule of a finitely-generated free $\Lambda$-module $M$. Then $N$ is free if and only if $M / N$ has no nonzero finite $\Lambda$-submodules.

Proof. Using Proposition 2.3 and the fact that $\Lambda$ is a local ring, we have that $N$ is free $\Leftrightarrow \operatorname{Ext}_{\Lambda}^{1}(N, L)=0$ for every $L \Leftrightarrow \operatorname{Ext}_{\Lambda}^{2}(M / N, L)=0$ for every $L \Leftrightarrow M / N$ has no finite $\Lambda$-submodules.

Corollary 2.5. Any pseudo-isomorphism between finitely-generated free $\Lambda$-modules is an isomorphism.

2.2. Tate twisting. Let $M$ be a $\Lambda$-module. The $n$-th Tate twist $M(n)$ has the same underlying $\mathbb{Z}_{p}$-module, but with the $\Gamma$-action twisted by the rule

$$
\gamma \cdot x=c(\gamma)^{n} \gamma x
$$

If $\mathbb{Z}_{p}$ has trivial $\Gamma$-action, then clearly

$$
M(n)=M \otimes_{\mathbb{Z}_{p}}\left(\mathbb{Z}_{p}(n)\right)
$$

as $\Lambda$-modules. Thinking of $\Lambda$ as the power series ring $\mathbb{Z}_{p}[[T]]$, we can interpret this twisting in another way. Given any automorphism $\phi$ of $\Lambda$-where we mean automorphism as topological ring - any module $M$ can be twisted to yield a new module $M_{\phi}$ in which $\lambda \cdot x=\phi(\lambda) x$. In particular, any linear substitution $T \mapsto c T+d$ with $c, d \in \mathbb{Z}_{p}, c$ a unit and $d=0 \bmod p$ defines such an automorphism. Among these we single out the case $c=c_{0}, d=c_{0}-1$. This automorphism will be denoted $\tau$ and called the Tate automorphism, the evident point being that $M(1)=M_{\tau}$.

Note that $\tau$ permutes the height one primes. In particular, we write $\tau_{n}=\tau^{n}(T)$. Written as an irreducible distinguished polynomial, $\tau_{n}=\left(T-\left(c_{0}^{n}-1\right)\right)$. These are the Tate primes, which will play an important role in the sequel.

2.3. Modules over $\Lambda^{\prime}$. Suppose first that $p$ is odd. Then the entire theory of finitely-generated $\Lambda$-modules extends in a straightforward way to $\Lambda^{\prime}$-modules. The point is that $\Lambda^{\prime}=\Lambda[\Delta]$, and $\Delta$ is finite of order prime to $p$. Hence $\Lambda^{\prime}$ splits as a direct product of topological rings into $p-1$ copies of $\Lambda$, and similarly for its category of modules. Explicitly, there are idempotents $e_{i} \in \mathbb{Z}_{p}[\Delta] \subset \Lambda^{\prime}, 0 \leq i \leq p-2$, such that for any module $M$ we have $M=\bigoplus e_{i} M$ as $\Lambda^{\prime}$-modules, with $\bar{\Delta}$ acting on $e_{i} M$ as $\omega^{i}$. Hence we can apply the structure theory to the summands $e_{i} M$ independently.

We also have $\operatorname{Spec} \Lambda^{\prime}=\coprod_{i=0}^{i=p-2} \operatorname{Spec} \Lambda$. If $q \in \operatorname{Spec} \Lambda$ and $n \in \mathbb{Z}$, we write $(q, n)$ for the prime $q$ in the $n$-th summand, $n$ of course being interpreted modulo $p-1$. Tate-twisting is defined as before, interpreting $\Lambda^{\prime}$ as $\mathbb{Z}_{p}\left[\left[\Gamma^{\prime}\right]\right]$. In terms of the product decomposition above, this means that $\tau^{\prime}$ permutes the factors by $(q, n) \mapsto(\tau(q), n+1)$. Hence there are Tate primes $\tau_{n}^{\prime}$ in $\Lambda^{\prime}$ defined by $\tau_{n}^{\prime}=\left(\tau_{n}, n\right)$.

If $p=2$, the situation is considerably more complicated, and indeed does not seem to be documented in the literature. The trouble is that now $\Delta$ has order 2 . Thus we still have that $\Lambda^{\prime}$ is a noetherian, profinite, local ring, but it does not split as a product of $\Lambda$ 's, and modules over it can have infinite projective dimension. See [16] for further discussion of the pseudo-isomorphism theory. 


\section{3. $p$-ADIC $K$-THEORY}

The spectra considered in this paper are usually $p$-complete, the only major exception being that we occasionally switch to an equivalent category of $p$-torsion spectra; see $\S 4.2$. Now if $Y$ is a $p$-complete spectrum, the functor $[-, Y]$ takes values in Ext-p-complete abelian groups. We review this category in $\S 3.1$; see [5], Ch. VI, $\S 2$ for further information and references. In $\S 3.2$ we recall some basic facts and notation concerning $p$-complete $K$-theory.

3.1. Ext-p-complete abelian groups. Recall that an abelian group $A$ is Ext- $p$ complete if

$$
\operatorname{Hom}\left(\mathbb{Z}\left[\frac{1}{p}\right], A\right)=0=\operatorname{Ext}\left(\mathbb{Z}\left[\frac{1}{p}\right], A\right) .
$$

The full subcategory $\mathcal{E}$ of Ext-p-complete abelian groups can also be described as the smallest abelian subcategory of the category $\mathcal{A}$ of all abelian groups that contains the $p$-complete groups. Here " $p$-complete" means that the natural map $A \longrightarrow \lim _{n} A / p^{n}$ is an isomorphism. Some further properties of Ext-p-complete groups are summarized in the following proposition:

Proposition 3.1. a) For any abelian group $A, \operatorname{Hom}\left(\mathbb{Z} / p^{\infty}, A\right)$ and $\operatorname{Ext}\left(\mathbb{Z} / p^{\infty}, A\right)$ are Ext-p-complete.

b) An Ext-p-complete group has no nonzero divisible subgroups.

c) The objects of $\mathcal{E}$ have a natural $\mathbb{Z}_{p}$-module structure.

d) $\mathcal{E}$ is closed under arbitary limits, and these are formed in $\mathcal{A}$.

e) If $A, B$ are Ext-p-complete, then so are $\operatorname{Hom}(A, B)$ and $\operatorname{Ext}(A, B)$.

The Ext-p-completion of an abelian group is $e A=\operatorname{Ext}\left(\mathbb{Z} / p^{\infty}, A\right)$.

Proposition 3.2. a) The Ext-p-completion functor is idempotent with image $\mathcal{E}$.

b) There is an exact sequence

$$
0 \longrightarrow D i v A \longrightarrow A \longrightarrow e A \longrightarrow \operatorname{Ext}\left(\mathbb{Z}\left[\frac{1}{p}\right], A\right) \longrightarrow 0,
$$

where Div $A$ is the maximal divisible subgroup of $A$.

c) There is a short exact sequence

$$
0 \longrightarrow \text { div } A \longrightarrow e A \longrightarrow \hat{A} \longrightarrow 0,
$$

where div $A$ is the subgroup of divisible elements. In particular, if $A$ has no nonzero divisible elements, then $e A$ is just the usual p-completion of $A$.

Here we recall that a group $B$ is divisible (meaning $p$-divisible, in our context) if $p B=B$. On the other hand, an element $x$ is divisible if $x=0 \bmod p^{n}$ for all $n$. For example, let $A$ be the abelian group with generators $x, y_{1}, y_{2}, \ldots$ and relations $p x=0, p^{n} y_{n}=x$. Then $A$ has no divisible subgroups, but its subgroup of divisible elements is a copy of $\mathbb{Z} / p$ generated by $x$. Note also that $A \longrightarrow e A$ is injective, and hence $e A$ is an Ext-p-complete group with nonzero divisible elements.

Note that $\mathcal{E}$ is not closed under arbitrary direct sums. For example, the direct sum of an infinite number of copies of $\mathbb{Z}_{p}$ is not Ext- $p$-complete. Nevertheless, the category $\mathcal{E}$ has its own intrinsic coproduct given by

$$
\coprod_{\alpha} A_{\alpha}=e\left(\bigoplus_{\alpha} A_{\alpha}\right) .
$$


In general, infinite coproduct functors on an abelian category need not be exact. For an interesting example directly related to our situation, see [11. So it is worth noting that coproducts are exact in $\mathcal{E}$.

Proposition 3.3. For any index set $I$, the functor $\left\{A_{\alpha}: \alpha \in I\right\} \mapsto \coprod_{\alpha} A_{\alpha}$ is exact.

Proof. The coproduct in $\mathcal{E}$ is formed in two stages: first we take the ordinary direct sum; this functor is exact. Then we apply Ext- $p$-completion, which is right exact with left derived functor $\operatorname{Hom}\left(\mathbb{Z} / p^{\infty},-\right)$. But if $B$ is any direct sum of Ext- $p$ complete groups, $\operatorname{Hom}\left(\mathbb{Z} / p^{\infty}, B\right)=0$. Hence the composite functor is exact.

Thus $\mathcal{E}$ also has all colimits, and these are created by first taking the colimit in $\mathcal{A}$ and then applying Ext-p-completion. Unfortunately, however, filtered colimits are not exact. In fact, even sequential colimits are only right exact. For example, consider the evident short exact sequence of direct systems

$$
0 \longrightarrow \mathbb{Z} / p \longrightarrow \mathbb{Z} / p^{n+1} \longrightarrow \mathbb{Z} / p^{n} \longrightarrow 0
$$

(in the first slot we have a sequence of $\mathbb{Z} / p$ 's connected by identity maps) whose colimit in $\mathcal{A}$ gives

$$
0 \longrightarrow \mathbb{Z} / p \longrightarrow \mathbb{Z} / p^{\infty} \longrightarrow \mathbb{Z} / p^{\infty} \longrightarrow 0
$$

Applying Ext-p-completion leaves the first term untouched while killing the other two.

Now suppose $M$ is a compact Hausdorff $\mathbb{Z}_{p}$-module, where as always we assume the module structure is continuous. Then $M$ is a profinite abelian $p$-group (cf. [17. Proposition 5.2.4) and hence is Ext-p-complete by Proposition [3.1]). Many Ext-p-complete groups do not have this form: for example, any infinite profinite group is uncountable, whereas any finite exponent group, whether countable or not, is Ext- $p$-complete. And of course no profinite group can have nonzero divisible elements.

Nevertheless, the category of compact Hausdorff $\mathbb{Z}_{p}$-modules and continuous homomorphisms admits a forgetful functor to Ext- $p$-complete abelian groups that will be exploited frequently in the sequel. One noteworthy point is that the continuous hom groups are still Ext-p-complete, because the group of continuous homomorphisms between two profinite modules is an inverse limit of finite exponent groups.

Finally, we recall that for any spectra $W, X$, there is a natural short exact sequence

$$
0 \longrightarrow \operatorname{Ext}\left(\mathbb{Z} / p^{\infty},[W, X]\right) \longrightarrow\left[W, X^{\wedge}\right] \longrightarrow \operatorname{Hom}\left(\mathbb{Z} / p^{\infty},\left[\Sigma^{-1} W, X\right]\right) \longrightarrow 0 .
$$

In particular, the hom-groups for $p$-complete spectra take values in $\mathcal{E}$.

3.2. $p$-adic topological K-theory. All spectra are implicitly completed at $p$; in particular, $K$ denotes the $p$-completion of the periodic complex $K$-theory spectrum. We will use the notation $K^{*} X=K^{0} X \oplus K^{1} X$. Then the ring of operations $K^{*} K$ is completely determined by $K \cdot K$ and has an elegant description in terms of the Iwasawa algebra ([14]; see also [15]).

Proposition 3.4. $K^{0} K$ is isomorphic to $\Lambda^{\prime}$. The isomorphism is uniquely determined by the correspondence $\psi^{k} \leftrightarrow \gamma$ with $c(\gamma)=k$ ( $k \in \mathbb{Z}$, p prime to $\left.k\right)$. Furthermore, $K^{1} K=0$. 
Using the idempotents $e_{i} \in \mathbb{Z}_{p}[\Delta]$, we obtain the classical splitting

$$
K \cong \bigvee_{i=0}^{p-2} e_{i} K
$$

Recall that the zero-th summand is itself a $(2 p-2)$-periodic ring spectrum, called the Adams summand and customarily denoted $E(1)$; furthermore, $e_{i} K \cong \Sigma^{2 i} E(1)$. Smashing with the Moore spectrum yields a similar decomposition, whose Adams summand $e_{0} K \wedge M \mathbb{Z} / p$ is usually denoted $K(1)$ - the first Morava K-theory.

Note also that $K^{0} S^{2 n}=\mathbb{Z}_{p}(n)$. More generally, $K^{0}\left(S^{2 n} \wedge X\right)=K^{0} X(n)$. Thus Tate twisting corresponds precisely to double suspension. Note also that the full ring of operations can be written as a twisted tensor product

$$
K^{*} K \cong \Lambda^{\prime} \tilde{\otimes} K^{*} S^{0}=\Lambda^{\prime} \tilde{\otimes} \mathbb{Z}_{p}\left[\beta, \beta^{-1}\right]
$$

where $\beta$ is the Bott class.

Let $\mathcal{N}=\Sigma^{-1} M \mathbb{Z} / p^{\infty}$. By a classical theorem of Adams [1, we have:

Proposition 3.5. There is a natural isomorphism

$$
K^{n} X \cong\left(K_{n-1} X \wedge \mathcal{N}\right)^{\#}
$$

where \# denotes Pontrjagin duality.

Corollary 3.6. The functors $K^{n} X$ take values in the category of compact $\Lambda^{\prime}$ modules and continuous homomorphisms.

Proof. The functor $X \mapsto K_{n-1}(X \wedge \mathcal{N})$ takes values in the category of discrete torsion $\Lambda^{\prime}$-modules. Pontrjagin duality then gives an equivalence from this category to the category of compact Hausdorff $\Lambda^{\prime}$-modules (see for example [17, Theorem 1.1.8).

In $\S 8.3$ we consider the general question of when $[-, Y]$ lifts to compact $\Lambda^{\prime}$ modules.

We conclude by recalling that for $p=2$, the operations in real $K$-theory can also be described in terms of the Iwasawa algebra.

Proposition 3.7. $K O^{0} K O \cong \Lambda$ and $K O^{*} K O \cong \Lambda \tilde{\otimes} K O^{*} S^{0}$.

\section{The $K(1)$-LOCAL CATEGORY}

This section reviews the stable homotopy category $L_{K(1)} \mathcal{S}$. We also introduce the notion of "quasi-small" object (§4.3), briefly discuss an equivalence of categories $L_{K} \mathcal{S}_{\text {tor }} \cong L_{K(1)} \mathcal{S}(\S 4.2)$, and review the $K$-based Adams spectral sequence (§4.6). The analysis of $L_{K(1)} \mathcal{S}$ is continued in more detail in later sections.

4.1. Smash products and units. $L_{K(1)} \mathcal{S}$ is a closed symmetric monoidal category with compatible triangulation (see [13, Appendix A for the definitions). It is important to note that the smash product of $p$-complete spectra need not be $p$-complete, and hence the smash product of two objects $X, Y$ in $L_{K(1)} \mathcal{S}$ is defined to be the $K(1)$-localization $L_{K(1)}(X \wedge Y)$. When no confusion can result, we will simply write $X \wedge Y$ for this intrinsic smash product (see $\S 4.2$ for the only exception to this convention). In contrast, the function spectrum $F(X, Y)$ is already $K(1)$-local. 
The unit object $L_{K(1)} S^{0}$ and the localized Moore spectrum $L_{K(1)} M \mathbb{Z} / p$ will similarly be written as $S^{0}, M \mathbb{Z} / p$, respectively. With this notation, there are fibre sequences in $L_{K(1)} \mathcal{S}$

$$
S^{0} \longrightarrow K \stackrel{T}{\longrightarrow} K, \quad S^{0} \longrightarrow K O \stackrel{T}{\longrightarrow} K O
$$

for $p$ odd, $p=2$, respectively.

In particular, we find that $S^{0}$ is in the thick subcategory generated by $K$. For $p=2$ this assertion depends on the well-known lemma:

Lemma 4.1. Suppose $f: \Sigma^{k} X \longrightarrow X$ is nilpotent. Then $X$ is in the thick subcategory generated by the cofibre $C_{f}$.

Applying this to $\eta: \Sigma K O \longrightarrow K O$, we have:

Proposition 4.2. $K O$ is in the thick subcategory generated by $K$.

This yields the $p=2$ case of our assertion above.

4.2. Complete spectra vs. torsion spectra. Let $\mathcal{S}_{\text {tor }}$ denote the full subcategory of $p$-torsion spectra. Then $L_{K}\left(\mathcal{S}_{t o r}\right)=\left(L_{K} \mathcal{S}\right)_{t o r}$, so we may use the notation $L_{K} \mathcal{S}_{\text {tor }}$ without ambiguity. Let $\mathcal{N}=\Sigma^{-1} M \mathbb{Z} / p^{\infty}$. Then there are mutually inverse equivalences of categories

$$
(-) \wedge \mathcal{N}: L_{K(1)} \mathcal{S} \longrightarrow L_{K} \mathcal{S}_{\text {tor }}, \quad F(\mathcal{N},-): L_{K} \mathcal{S}_{\text {tor }} \longrightarrow L_{K(1)} \mathcal{S}
$$

These are equivalences of stable homotopy categories in the evident sense, preserving unit objects, coproducts, smash products, etc. Note that in $L_{K} \mathcal{S}_{\text {tor }}$, smash products and coproducts are formed in $\mathcal{S}$, while products and function objects have to be reflected back by smashing with $\mathcal{N}$ - exactly the opposite of what happens in $L_{K(1)} \mathcal{S}$. See [16] for further discussion.

Note: When working in $L_{K} \mathcal{S}_{\text {tor }}$, the smash product is always the ordinary smash product. Our convention that $X \wedge Y$ means $L_{K(1)}(X \wedge Y)$ only applies when $X, Y \in L_{K(1)} \mathcal{S}$.

4.3. Coproducts. The category $L_{K(1)} \mathcal{S}$ also has arbitrary coproducts, obtained by $K(1)$-localizing the wedge. In this case the distinction between the two "wedges" comes up frequently enough to warrant a separate notation:

$$
\coprod_{\alpha} X_{\alpha}=L_{K(1)}\left(\bigvee_{\alpha} X_{\alpha}\right) \text {. }
$$

This dichotomy leads to two possible definitions of "small" object in $L_{K(1)} \mathcal{S}$. Following [13, we reserve the term small for objects $W$ such that the natural map

$$
\bigoplus_{\alpha}\left[W, X_{\alpha}\right] \longrightarrow\left[W, \coprod_{\alpha} X_{\alpha}\right]
$$

is an isomorphism for arbitrary collections $X_{\alpha}$. We call $W$ quasi-small if the natural map

$$
e\left(\bigoplus_{\alpha}\left[W, X_{\alpha}\right]\right) \longrightarrow\left[W, \coprod_{\alpha} X_{\alpha}\right]
$$

is an isomorphism. For example, $M \mathbb{Z} / p$ is small, whereas $S^{0}$ is only quasi-small. To see that $S^{0}$ is quasi-small, note that since the $X_{\alpha}$ 's are $p$-complete,

$$
\operatorname{Hom}\left(\mathbb{Z} / p^{\infty}, \bigoplus \pi_{-1} X_{\alpha}\right)=0
$$


Hence

$$
e\left(\bigoplus \pi_{0} X_{\alpha}\right) \cong \pi_{0}\left(L_{K(1)}\left(\bigvee X_{\alpha}\right)\right)=\pi_{0}\left(\coprod X_{\alpha}\right)
$$

On the other hand, $\bigoplus \pi_{0} X_{\alpha}$ need not be $p$-complete; for example, $\bigoplus_{n=1}^{\infty} \pi_{0} S^{0}=$ $\bigoplus_{n=1}^{\infty} \mathbb{Z}_{p}$ is not. Hence $S^{0}$ is not small. We will consider this issue more systematically in later sections.

4.4. Localizing subcategories. Now recall that a subcategory is said to be localizing if it is thick and closed under arbitrary coproducts. The following important fact is a special case of [12, Theorem 7.5.

Theorem 4.3. $L_{K(1)} \mathcal{S}$ has no proper nontrivial localizing subcategories.

This theorem implies that a natural transformation of cohomology theories is an isomorphism provided that is an isomorphism on a single object.

The proof involves a result of independent interest on cellular towers. Let $A$ be a collection of objects of $L_{K(1)} \mathcal{S}$ and let $\Sigma^{*} A$ denote the collection obtained by adjoining all suspensions of objects of $A$. An $A$-cellular tower is a sequence $X_{0} \longrightarrow X_{1} \longrightarrow X_{2} \longrightarrow \ldots$ such that the cofibre of each map $X_{n} \longrightarrow X_{n+1}$ is a coproduct of elements of $\Sigma^{*} A$. We say that a given $X$ admits an $A$-cellular tower if there exists an $A$-cellular tower as above and an equivalence

$$
\text { hocolim }_{n} X_{n} \stackrel{\cong}{\longrightarrow} X \text {. }
$$

Here the sequential homotopy colimit hocolim $_{n} X_{n}$ is defined as the usual telescope, formed as the cofibre the appropriate map $\amalg X_{n} \longrightarrow \coprod X_{n}$.

Lemma 4.4. Any $X$ in $L_{K(1)} \mathcal{S}$ admits an $M \mathbb{Z} / p$-cellular tower.

The lemma follows from an extremely general result ([13, Theorem 2.3.2). What this reference actually proves is the following: let $\mathcal{C}$ be any triangulated category with arbitrary coproducts (so small objects and sequential "homotopy colimits" can be defined as above). Then $A$-cellular towers can be defined in the evident way, and we have:

Lemma 4.5. Suppose $A$ is a set of small objects in $\mathcal{C}$ with the property that if $Z$ is an object of $\mathcal{C}$ and $[W, Z]=0$ for all $W \in \Sigma^{*} A$, then $Z=*$.

Then any object of $\mathcal{C}$ admits an A-cellular tower.

Thus Lemma 4.4 is a special case. Now let $\underline{l o c} X$ denote the smallest localizing subcategory containing the object $X$. Then Lemma 4.4 yields the corollary:

Corollary 4.6. $\underline{\operatorname{loc}} M \mathbb{Z} / p=L_{K(1)} \mathcal{S}=\underline{\operatorname{loc}} S^{0}$.

It follows from the second equality above that any localizing subcategory is an ideal. Now suppose $\mathcal{D}$ is a localizing subcategory containing a nontrivial object $X$. Then $K(1) \wedge X \in \mathcal{D}$ and hence $K(1) \in \mathcal{D}$. It follows that $M \mathbb{Z} / p \in \mathcal{D}$, using Proposition 4.2 in the case $p=2$. Hence $\mathcal{D}=L_{K(1)} \mathcal{S}$ by the corollary. This proves Theorem 4.3,

4.5. $L_{K(1)} \mathcal{S}$ as stable homotopy category. Recall from [13] the definition of an algebraic stable homotopy category: This means a category $\mathcal{C}$ such that

(i) $\mathcal{C}$ is a triangulated category with a compatible closed symmetric monoidal structure;

(ii) $\mathcal{C}$ has arbitrary coproducts; 
(iii) cohomology functors are representable;

(iv) there is a set $A$ of small objects such that the only localizing subcategory containing $A$ is $\mathcal{C}$.

Theorem 4.7. $L_{K(1)} \mathcal{S}$ is an algebraic stable homotopy category.

Here we take $A=\left\{\Sigma^{*} M \mathbb{Z} / p\right\}$. Item (iii), Brown representability, follows from the corresponding fact in $\mathcal{S}$ or by a direct argument. The remaining items have already been discussed above.

Note that a cohomology functor $\mathfrak{h}^{*}$ must take values in the Ext-p-complete abelian groups $\mathcal{E}$, since it is representable. This also follows directly from Lemma4.4 since $\mathcal{E}$ is closed under products.

Dually, any homology functor $\mathfrak{h}_{*}: L_{K(1)} \mathcal{S} \longrightarrow \mathcal{A}$ takes values in $p$-torsion groups. This again follows from Lemma 4.4 since a homology functor by definition takes coproducts to direct sums. Hence it is not surprising that Brown representability for homology functors takes the following form (12, Theorem 9.5).

Theorem 4.8. Any homology functor $\mathfrak{h}: L_{K(1)} \mathcal{S} \longrightarrow \mathcal{A}$ is representable in the sense that there is an object $Y \in L_{K(1)} \mathcal{S}$ and a natural isomorphism

$$
\mathfrak{h}(X) \cong \pi_{0}(X \wedge Y \wedge \mathcal{N}) \text {. }
$$

Furthermore, any natural transformation $\mathfrak{h} \longrightarrow \mathfrak{h}^{\prime}$ between two such functors is induced by a map $Y \longrightarrow Y^{\prime}$ of the representing objects, unique up to phantom maps.

One can also consider $\mathcal{E}$-valued homology functors; that is, exact functors $\mathfrak{h}$ : $L_{K(1)} \mathcal{S} \longrightarrow \mathcal{E}$ that commute with arbitrary coproducts. Any object $E \in L_{K(1)} \mathcal{S}$ defines such a homology functor in the usual way: $\mathfrak{h}(X)=\pi_{0}(E \wedge X)$, using the intrinsic smash product. However, these functors need not commute with filtered or even sequential colimits of small objects (see [11, or the example in §3.1), which limits their utility.

4.6. The $K$-based Adams spectral sequence. The $K$-based Adams spectral sequence is discussed in [6], [12] and, in the form most directly relevant to this paper, [16]. It has $E_{2}$-term

$$
E_{2}^{s, t}=\operatorname{Ext}_{\Lambda^{\prime}}^{s}\left(K^{\cdot} X, K^{\cdot} \Sigma^{t} Y\right)
$$

and converges to $\left[\Sigma^{t-s} Y, X\right]$. The $E x t$-groups are to be computed in the category of compact topological $\Lambda^{\prime}$-modules (see [17] for a general discussion of compact modules over pro-group rings). Continuous Pontrjagin duality gives an isomorphism between this category and the category of discrete $p$-torsion $\Lambda^{\prime}$-modules; furthermore, this correspondence fits perfectly with the equivalence of stable homotopy categories $L_{K(1)} \mathcal{S} \cong L_{K} \mathcal{S}_{\text {tor }}$, using Proposition 3.5. Hence it is very easy to move back and forth between $L_{K(1)} \mathcal{S}$ and compact $\Lambda^{\prime}$-modules on the one hand, and $L_{K} \mathcal{S}_{\text {tor }}$ and discrete torsion $\Lambda^{\prime}$-modules on the other.

In fact the convergence of the spectral sequence is uniform, in the following sense: there is a $d \geq 0$ such that the spectral sequence collapses at $E_{d}$ and the $d$-th Adams filtration vanishes. When $p$ is odd, $d=3$ and in fact the entire spectral sequence is concentrated in $s=0,1,2$.

When $p=2$ one can take $d=6$; cf. [16. (Is this sharp? The examples we know have $d$ at most 5.) We note the following special cases with $p=2$ :

1. Suppose $K \cdot X$ is acyclic as a $\mathbb{Z}_{2}[\Delta]$-module. Then by Shapiro's lemma, $E_{2}^{s, t}=$ 0 for $s>2$. In other words, this case behaves formally like the odd primary case, 
with $d=3$. This remark applies to any $X$ after smashing with the mapping cone of $\eta$, since $K^{0} C_{\eta}=\mathbb{Z}_{2}[\Delta]$ as a $\mathbb{Z}_{2}[\Delta]$-module and $K^{1} C_{\eta}=0$, and hence

$$
K \cdot\left(X \wedge C_{\eta}\right) \cong K \cdot X \otimes_{\mathbb{Z}_{2}} K^{\cdot} C_{\eta} .
$$

Note the module on the right is $\Delta$-acyclic.

2. Take $X=K O$. Then another application of Shapiro's lemma shows that

$$
E_{2}^{s, t}=H^{s}\left(\Delta ; K^{0} \Sigma^{t} Y\right) .
$$

In this case the convergence is uniform with $d=4$. To see this, note that $K O$ admits an Adams tower in which each term is a suspension of $K O$, the cofibres are suspensions of $K$ and the connecting maps in the tower are all multiplication by $\eta$. In fact this is the Adams tower associated to the standard periodic resolution of the $\Lambda[\Delta]$-module $\Lambda$. Since $\eta^{3}=0$ in $\pi_{*} K O$, this proves our claim.

\section{Dualizable objects}

An object $X \in L_{K(1)} \mathcal{S}$ is dualizable if the natural map $D X \wedge Y \longrightarrow F(X, Y)$ is an equivalence. Here $D X=F\left(X, S^{0}\right)$, and the smash product is the intrinsic smash product, as usual. For a convenient summary of basic facts about dualizable objects in symmetric monoidal categories, see [13], Appendix A2. For example, $X$ is dualizable if and only if the the more general dualizing map

$$
F(X, Z) \wedge Y \longrightarrow F(X, Z \wedge Y)
$$

is an equivalence for all $Y, Z$.

An object $X$ is $F$-small if the natural map

$$
\coprod_{\alpha} F\left(X, Y_{\alpha}\right) \longrightarrow F\left(X, \coprod_{\alpha} Y_{\alpha}\right)
$$

is an equivalence for arbitrary collections $Y_{\alpha}$ ([13], Definition 2.1.1).

The next theorem (except for part c)) is a special case of [12], Theorem 8.6.

Theorem 5.1. The following are equivalent:

a) $X$ is dualizable,

b) $X$ is F-small,

c) $X$ is quasi-small,

d) $K(1)_{*} X$ is finite-dimensional over $K(1)_{*}$,

e) $K \cdot X$ is finitely-generated over $\mathbb{Z}_{p}$.

Proof. (a) $\Leftrightarrow$ (b) is valid in any algebraic stable homotopy category; see [13], Theorem 2.1.3c.

(b) $\Leftrightarrow\left(\right.$ c) is straightforward from the definitions: since $S^{0}$ is quasi-small, as noted earlier, the induced map on $\pi_{0}$

$$
\pi_{0}\left(\coprod_{\alpha} F\left(X, Y_{\alpha}\right)\right) \longrightarrow \pi_{0} F\left(X, \coprod_{\alpha} Y_{\alpha}\right)
$$

has the form

$$
e\left(\bigoplus_{\alpha}\left[X, Y_{\alpha}\right]\right) \longrightarrow\left[X, \coprod_{\alpha} Y_{\alpha}\right]
$$

and similarly for the other homotopy groups. Hence this map is an isomorphism if and only if $X$ is quasi-small.

(d) $\Leftrightarrow($ e) follows from Nakayama's lemma. 
It remains to show $(\mathrm{a}) \Leftrightarrow(\mathrm{d})$. In our special case, the proof in 12 can be summarized in the following diagram, in which $K(1)$ has been abbreviated as $\kappa$ :

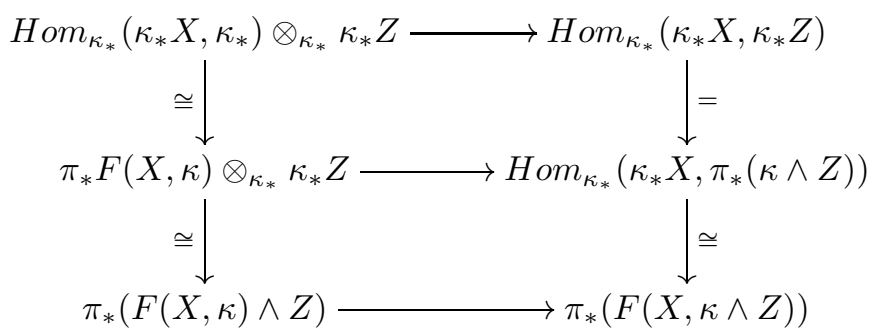

The vertical maps are isomorphisms by general properties of module spectra (cf. [12], Proposition 3.4). If $X$ is dualizable, then the bottom map is an isomorphism and hence so is the top map. But the latter map has the form $V^{*} \otimes$ $W \longrightarrow \operatorname{Hom}(V, W)$ as vector spaces over $\kappa_{*}$, forcing $\operatorname{dim}_{\kappa_{*}} \kappa_{*} X<\infty$.

Conversely, if $\operatorname{dim}_{\kappa_{*}} \kappa_{*} X<\infty$, then the top map is an isomorphism and hence so is the bottom map. In other words, the dualizing map $F(X, Y) \wedge Z \longrightarrow F(X, Y \wedge Z)$ is an equivalence when $Y=\kappa$. But the collection of all $Y$ for which the dualizing map is an equivalence is a thick subcategory, and the thick subcategory generated by $\kappa$ contains $M$ (see $\S 4.1)$. We conclude that $D X \wedge Y \longrightarrow F(X, Y)$ is an equivalence after smashing with $M$, and hence is an equivalence.

\section{Small obJeCts}

Recall that an object $X \in L_{K(1)} \mathcal{S}$ is small if the natural map $\bigoplus_{\alpha}\left[X, Y_{\alpha}\right] \longrightarrow$ $\left[X, \coprod Y_{\alpha}\right]$ is an isomorphism, for arbitrary coproducts $\coprod Y_{\alpha}$. In this section we characterize the small objects ([12], Theorem 8.5).

Theorem 6.1. The following are equivalent:

a) $X$ is small,

b) $X$ is $M \mathbb{Z} / p$-finite,

c) $X$ is the $K(1)$-localization of a finite p-torsion spectrum,

d) $K \cdot X$ is finite,

e) $X$ is dualizable and of finite exponent.

Proof. $(\mathrm{a}) \Rightarrow(\mathrm{b})$ : This follows from a general result valid in any algebraic stable homotopy category; see [13], Corollary 2.3.12.

(b) $\Rightarrow(\mathrm{c})$ : See [3]. The key point is that the $K(1)$-localization of a finite $p$ torsion spectrum $F$ can be constructed as the homotopy colimit of a sequence $F=F_{0} \longrightarrow F_{1} \longrightarrow F_{2} \longrightarrow \ldots$ in which each $F_{n}$ is a finite $p$-torsion spectrum and each map is a $K(1)$-equivalence. Hence maps between localizations of finite $p$-torsion spectra can be realized as localizations of maps between two other such spectra. This makes it possible to induct on the "length" of an $M \mathbb{Z} / p$-finite spectrum $X$.

(c) $\Rightarrow(d)$ : This is immediate.

(d) $\Rightarrow(\mathrm{e})$ : Since $K^{\cdot} X$ is finitely-generated over $\mathbb{Z}_{p}, X$ is dualizable. Since $K \cdot X$ is finite, $p^{m}$ annihilates it for some $m$. Then $p^{m}$ has Adams filtration one and so is nilpotent.

$(\mathrm{e}) \Rightarrow(\mathrm{a})$ : By assumption $p^{m}$ annihilates $X$ for some $m$; hence $X$ is a retract of $X \wedge M \mathbb{Z} / p^{m}$. An easy formal argument shows that the smash product of a dualizable object and a small object is small ([13], Theorem 2.1.3a). Hence $X$ is small. 
The key lemma of Bousfield used in the proof of $(b) \Rightarrow(c)$ actually shows more. We record the more general statement here for future reference.

Proposition 6.2. Suppose $F_{1}, F_{2}$ are finite spectra at least one of which is $p$ torsion. Then the cofibre of any map $L_{K(1)} F_{1} \longrightarrow L_{K(1)} F_{2}$ is the localization of a finite spectrum.

The category of small objects is irreducible, in the following sense (see [12, Theorem 6.9).

Theorem 6.3. The thick subcategory of small objects has no proper nontrivial thick subcategories.

See the original paper of Hopkins (9, Theorem 7) for the proof. Since we are working in $L_{K(1)} \mathcal{S}$, everything is of course much easier. In particular, the nilpotence theorem reads as follows.

Proposition 6.4. If $X$ has finite exponent and $f: \Sigma^{k} X \longrightarrow X$ induces the zero homomorphism on $K(1)_{*}$, then $f$ is nilpotent.

In $L_{K(1)} \mathcal{S}$ this is easy because of the uniform vanishing line for the $K^{*}$-Adams spectral sequence. The theorem is then deduced as in $[9]$.

\section{INVERTIBLE OBJECTS}

This section is a summary and translation of some results from 10 .

Recall that an object $X$ is invertible if there is an object $Y$ such that $X \wedge Y \cong S^{0}$. Here the smash product is the intrinsic smash product in $L_{K(1)} \mathcal{S}$, as usual. In fact, a formal argument shows that if $Y$ exists, then $Y \cong D X([13$, A.2.8). The set of isomorphism classes of invertible objects forms a group under smash product: the Picard group, denoted Pic $L_{K(1)} \mathcal{S}$.

Proposition 7.1. $X$ is invertible if and only if $K \cdot X$ is free of rank one as a $\mathbb{Z}_{p}$-module.

In particular, only one of $K^{0} X, K^{1} X$ can be nonzero. To simplify the discussion we will assume $K^{1} X=0$. The collection of all such $X$ forms a subgroup of Pic $L_{K(1)} \mathcal{S}$, denoted $P i c^{0} L_{K(1)} \mathcal{S}$. Note that there is a natural homomorphism

$$
\phi: P i c^{0} L_{K(1)} \mathcal{S} \longrightarrow P i c \Lambda^{\prime},
$$

where Pic $\Lambda^{\prime}$ is the group of $\Lambda^{\prime}$-modules invertible under tensor product. Note further that

$$
\operatorname{Pic} \Lambda^{\prime} \cong \operatorname{Hom}\left(\Gamma^{\prime}, \mathbb{Z}_{p}^{\times}\right) \cong \begin{cases}\mathbb{Z}_{p}^{\times} & \text {if } p \text { odd } \\ \mathbb{Z}_{2}^{\times} \times \mathbb{Z} / 2 & \text { if } p=2 .\end{cases}
$$

To see this, note that $M$ is invertible if and only if it is free of rank one as a $\mathbb{Z}_{p^{-}}$ module; in other words, it is a rank one representation of $\Gamma^{\prime}$. Such representations are in bijective correspondence with $\operatorname{Hom}\left(\Gamma^{\prime}, \mathbb{Z}_{p}^{\times}\right)$. Since $\Gamma^{\prime}$ is itself isomorphic to $\mathbb{Z}_{p}^{\times}$, this yields our claim.

Proposition 7.2. For $p$ odd, $\phi$ is an isomorphism $\operatorname{Pic}^{0} L_{K(1)} \mathcal{S} \stackrel{\cong}{\cong}$ Pic $\Lambda^{\prime}$. 
That $\phi$ is injective is immediate from the Adams spectral sequence. In more detail: note that for any $K(1)$-local spectrum $X$ with $K \cdot X$ of projective dimension at most one, the Adams spectral sequence for maps into $X$ collapses. Hence $X$ is determined up to a noncanonical equivalence by the $\Lambda^{\prime}$-module $K \cdot X$. When $p$ is odd, any invertible spectrum has $K^{\cdot} X$ of projective dimension one by Proposition 7.1 and Proposition 2.2

The surjectivity is also immediate, using fibres of suitable self-maps $e_{i} K \longrightarrow e_{i} K$. Let $\mathbb{Z}_{p}^{(1)}$ denote the $p$-adic units congruent to one $\bmod p$. Then explicitly, we have

$$
\operatorname{Hom}\left(\Gamma^{\prime}, \mathbb{Z}_{p}^{\times}\right)=\operatorname{Hom}\left(\Gamma, \mathbb{Z}_{p}^{(1)}\right) \times \operatorname{Hom}\left(\Delta, \mu_{p-1}\right) .
$$

Given $\chi: \Gamma \longrightarrow \mathbb{Z}_{p}^{(1)}$ and $\omega^{i}: \Delta \longrightarrow \mu_{p-1}$, the fibre of $\gamma_{0}-\chi\left(\gamma_{0}\right): e_{i} K \longrightarrow e_{i} K$ realizes the corresponding invertible $\Lambda^{\prime}$-module. This translates into our Iwasawamodule terminology as follows: a rank one representation of $\Gamma$ corresponds to a $\Lambda$ module $\Lambda / f$, where $f=T-a$ is a linear distinguished polynomial. Since $\gamma_{0}=1+T$, $a=\chi\left(\gamma_{0}\right)-1$. We let $X_{f, i}$ denote the fibre of $f: e_{i} K \longrightarrow e_{i} K$.

When $p=2$ we have

$$
\operatorname{Hom}\left(\Gamma^{\prime}, \mathbb{Z}_{2}^{\times}\right)=\operatorname{Hom}\left(\Gamma^{(1)}, \mathbb{Z}_{2}^{\times}\right) \times \operatorname{Hom}\left(\Delta, \mathbb{Z}_{2}^{\times}\right)=\mathbb{Z}_{2}^{\times} \times\{ \pm 1\} .
$$

Then the element $(\chi, 1)$ of $P i c \Lambda^{\prime}$ is realized by the fibre of $\gamma_{0}-\chi\left(\gamma_{0}\right): K O \longrightarrow K O$, while the element $(1,-1)$ is realized by the cofibre $Y$ of $S^{0} \longrightarrow S_{\mathbb{C}}^{0}$. Here $S_{\mathbb{C}}^{0}$ the "complexified sphere" - is just the fibre of $T: K \longrightarrow K$. This shows that $\phi$ is surjective for $p=2$ also. The kernel, however, is nontrivial. For $f \in \Lambda$, let $X_{f}^{\mathbb{R}}$ denote the fibre of $f: K O \longrightarrow K O$. Now take

$$
W=\Sigma^{-4} X_{(T-24)}^{\mathbb{R}} .
$$

Note that with our convention that $\gamma_{0}=\psi^{5}, T-24$ corresponds to $\psi^{5}-25$. Thus $K^{0} X_{(T-24)}^{\mathbb{R}}=\mathbb{Z}_{2}(2)$, and $X_{(T-24)}^{\mathbb{R}}$ is a "fake $S^{4}$ ".

Lemma 7.3. Suppose $K \cdot X \cong K \cdot S^{0}$ as $\Lambda^{\prime}$-modules. Then either

(a) $X \cong S^{0}$ (if $K O^{0} X \stackrel{\cong}{\longrightarrow} K^{0} X$ ) or

(b) $X \cong W$ (if $K O^{-4} X \stackrel{\cong}{\longrightarrow} K^{-4} X$ ).

The alternatives correspond to the two possible $d_{3}$ 's in the Adams spectral sequence converging to $K O^{*} X$, as can be seen on inspection. Furthermore, these alternatives are exclusive, and $W$ is not equivalent to $S^{0}$.

Theorem 7.4. (a) There is a short exact sequence

$$
0 \longrightarrow \mathbb{Z} / 2 \longrightarrow P i c^{0} L_{K(1)} \mathcal{S} \longrightarrow P i c \Lambda^{\prime} \longrightarrow 0,
$$

with the kernel $\mathbb{Z} / 2$ generated by $W$.

(b) $Y \wedge Y \cong W$. Hence $Y$ has order four, the sequence is nonsplit, and

$$
\operatorname{Pic}{ }^{0} L_{K(1)} \mathcal{S} \cong \mathbb{Z}_{2} \times \mathbb{Z} / 2 \times \mathbb{Z} / 4 \text {. }
$$

(c) $W \cong L_{K(1)} C_{\zeta}$, where $\zeta: \Sigma M \mathbb{Z} / 2 \longrightarrow S^{0}$ is one of the two maps of order four.

The theorem follows from the lemma, by direct computation. The generators for the three factors in (b) can be taken respectively as $X_{T-4}^{\mathbb{R}}, X_{T+2}^{\mathbb{R}}$, and $Y$. Note that the $\mathbb{Z}_{2} \times \mathbb{Z} / 2$ part corresponds to those invertible $X$ which can be realized as the fibre of a map $\mathrm{KO} \longrightarrow K O$. 
Given any $E \in L_{K(1)} \mathcal{S}$, we define the inertia group $I_{E} \subset P i c^{0} L_{K(1)} \mathcal{S}$ by

$$
I_{E}=\left\{X \in P i c^{0} L_{K(1)} \mathcal{S}: E \wedge X \cong E\right\} .
$$

For example, $I_{S^{0}}=\{1\}$ and $I_{K}=P i c^{0} L_{K(1)} \mathcal{S}$. A more interesting example:

Proposition 7.5. Let $p=2$. Then $I_{K O}=\mathbb{Z}_{2} \times \mathbb{Z} / 2$ as in Proposition 7.4b). The element $Y$ of order 4 acts by $K O \wedge Y \cong K O \wedge S^{2}$. Hence $K O \wedge W \cong \Sigma^{4} K O \cong$ $\Sigma^{-4} K O$.

Proof. Suppose the invertible object $X$ lies in the subgroup $\mathbb{Z}_{2} \times \mathbb{Z} / 2$, so there is a fibre sequence $X \stackrel{i}{\longrightarrow} K O \stackrel{f}{\longrightarrow} K O$. Then $K^{0} X \cong \mathbb{Z}_{2}$ is generated by the composite $X \stackrel{i}{\longrightarrow} K O \stackrel{c}{\longrightarrow} K$, where $c$ is complexification. Hence

$$
K^{0}(K O \wedge X) \cong K^{0} K O \otimes_{\mathbb{Z}_{2}} K^{0} X \cong \Lambda
$$

as a $\Lambda^{\prime}$-module, generated by the composite

$$
K O \wedge X \stackrel{c \wedge c i}{\longrightarrow} K \wedge K \longrightarrow K .
$$

Since $c$ is a ring map, we can rewrite this composite as

$$
K O \wedge X \stackrel{1 \wedge i}{\longrightarrow} K O \wedge K O \stackrel{m}{\longrightarrow} K O \stackrel{c}{\longrightarrow} K .
$$

This shows that $m \circ(1 \wedge i)$ induces an isomorphism $\Lambda=K^{0} K O \cong K^{0}(K O \wedge X) \cong \Lambda$. Since $K^{1} K O=0=K^{1}(K O \wedge X), m \circ(1 \wedge i)$ is an equivalence. This proves that $\mathbb{Z}_{2} \times \mathbb{Z} / 2 \subset I_{K O}$.

Now consider the fibre sequence $K O \wedge S^{0} \longrightarrow K O \wedge S_{\mathbb{C}}^{0} \longrightarrow K O \wedge Y$. Since

$$
K^{0}\left(K O \wedge S_{\mathbb{C}}^{0}\right) \cong K^{0} K O \otimes_{\mathbb{Z}_{2}} \mathbb{Z}_{2}[\Delta] \cong \Lambda^{\prime}
$$

$K O \wedge S_{\mathbb{C}}^{0} \cong K$. Furthermore, the map $K O \wedge S^{0} \longrightarrow K O \wedge S_{\mathbb{C}}^{0}$ can be identified with $c: K O \longrightarrow K$, which has cofibre $\Sigma^{2} K O$. This completes the proof of Proposition 7.5.

\section{8. $K$-Finite AND WEAKLY DUALIZABLE OBJECTS}

In this section we study the thick subcategory of $L_{K(1)} \mathcal{S}$ consisting of those $X$ such that $K^{\prime} X$ is finitely-generated over $\Lambda$, or equivalently over $\Lambda^{\prime}$. The following theorem summarizes the main results.

Theorem 8.1. The following are equivalent:

a) $K \cdot X$ is a finitely-generated $\Lambda$-module,

b) $X$ is $K$-finite,

c) $\pi_{n} X$ is finitely-generated over $\mathbb{Z}_{p}$ for all $n$,

d) $X$ is weakly dualizable,

e) $X$ is weakly Brown-Comenetz dualizable,

f) The natural topology on $[-, X]$ is compact.

Except for part c), most of this appears in a more general context in [12]; see especially Theorem 10.2 there. The proof of the theorem above is divided into several steps, which appear below as Theorem 8.2. Proposition 8.10, Theorem 8.11, and Proposition 8.17.

We also give a universal coefficient spectral sequence for the $K$-theory of the Brown-Comenetz dual (Theorem 8.19).

The thick subcategory satisfying these equivalent conditions will be denoted $L_{K(1)}^{f i n} \mathcal{S}$. 
8.1. $K$-finite spectra. An object $X \in L_{K(1)} \mathcal{S}$ is $K$-finite if it is in the thick subcategory generated by $K$.

Theorem 8.2. The following are equivalent:

a) $K \cdot X$ is finitely-generated over $\Lambda$,

b) $X$ is $K$-finite,

c) $\pi_{n} X$ is a finitely-generated $\mathbb{Z}_{p}$-module for all $n \in \mathbb{Z}$.

Proof. (a) $\Rightarrow(\mathrm{b})$ : Suppose first that $p$ is odd. Then $X$ has an Adams resolution of length two

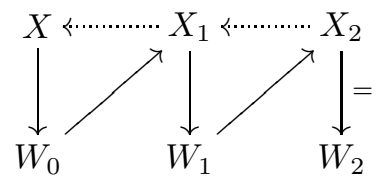

in which each $W_{i}$ is $K$-finite. Here we have used the fact that $\Lambda$ is noetherian. Hence $X$ is also $K$-finite.

Now suppose $p=2$. If $K \cdot X$ if $\Delta$-acylic, then it has projective dimension at most two over $\Lambda^{\prime}$, and hence the argument used for $p$ odd still applies. In particular, it follows that $X \wedge C_{\eta}$ is $K$-finite. Then Lemma 4.1 completes the proof.

(b) $\Rightarrow$ (c): This is immediate.

(c) $\Rightarrow$ (a): For this we will use an algebraic lemma.

Lemma 8.3. Let $M$ be a compact $\Lambda$-module with $p^{k} M=0$, and suppose that $\operatorname{Ext}_{\Lambda}^{1}\left(M, \mathbb{Z} / p^{k}\right)$ is a finitely-generated (and hence finite) $\mathbb{Z}_{p}$-module. Then $M$ is a finitely-generated $\Lambda$-module.

Proof. By a general result on modules over compact group-rings ([17, Corollary 5.2 .9 p. 232), we have a natural isomorphism

$$
\left(\operatorname{Ext}_{\Lambda}^{1}\left(M, \mathbb{Z} / p^{k}\right)\right)^{\#} \cong \operatorname{Tor}_{1}^{\Lambda}\left(M, \mathbb{Z} / p^{k}\right)
$$

Here $(-)^{\#}$ denotes Pontrjagin dual. For any compact $M$ we can compute the Tor $_{1}$ using the standard resolution of $\mathbb{Z} / p^{k}$ :

$$
0 \longrightarrow \Lambda \stackrel{i}{\longrightarrow} \Lambda \oplus \Lambda \stackrel{j}{\longrightarrow} \Lambda \longrightarrow \mathbb{Z} / p^{k} \longrightarrow 0,
$$

where $i(a)=\left(T a, p^{k} a\right)$ and $j(x, y)=p^{k} x-T y$. We find that

$$
\operatorname{Tor}_{1}^{\Lambda}\left(M, \mathbb{Z} / p^{k}\right) \cong\left\{(x, y) \in M \oplus M: p^{k} x=T y\right\} /\left\{\left(T z, p^{k} z\right)\right\} .
$$

When $p^{k} M=0$, this is just $M / T M \oplus\{y \in M: T y=0\}$. Hence if the $\operatorname{Ext}^{1}$ is finite, so is $M / T M$ and hence so is $M / \mathfrak{m} M$. The topological Nakayama lemma (see for example [17, Ch.V,$\S 2$, exercise 6) then implies that $M$ is a finitely-generated $\Lambda$-module.

Now suppose that $\pi_{n} X$ is finitely-generated over $\mathbb{Z}_{p}$ for every $n$. We want to show that $K \cdot X$ is finitely-generated over $\Lambda$. By Nakayama's lemma, it is enough to show that $K^{\cdot} X / p$ is finitely-generated over $\Lambda$. Hence it is enough to show that $K \cdot(X \wedge M \mathbb{Z} / p)$ is finitely-generated over $\Lambda$. Note that

(i) $p \cdot K \cdot(X \wedge M \mathbb{Z} / p)=0($ even when $p=2)$,

and

(ii) $\left[\Sigma^{k} M \mathbb{Z} / p, X \wedge M \mathbb{Z} / p\right]$ is finite for all $k$. 
Now suppose that $p$ is odd. Then since $E_{2}^{1, *}=E_{\infty}^{1, *}$ in the Adams spectral sequence, by (ii) we have that $\operatorname{Ext}_{\Lambda^{\prime}}^{1}\left(K^{\cdot}(X \wedge M \mathbb{Z} / p), \mathbb{Z} / p(n)\right)$ is finite for all $n$. Since

$$
\operatorname{Ext}_{\Lambda^{\prime}}^{1}\left(K^{\cdot}(X \wedge M \mathbb{Z} / p), \mathbb{Z} / p(n)\right)=\operatorname{Ext}_{\Lambda}^{1}\left(e_{i} K^{\cdot}(X \wedge M \mathbb{Z} / p), \mathbb{Z} / p\right)
$$

for $i=n \bmod p-1$, we conclude that $\operatorname{Ext}_{\Lambda}^{1}\left(K^{\cdot}(X \wedge M \mathbb{Z} / p), \mathbb{Z} / p\right)$ is finite. It then follows from Lemma 8.3 that $K^{\cdot}(X \wedge M \mathbb{Z} / p)$ is a finitely-generated $\Lambda$-module.

Finally, suppose $p=2$. If $K^{\cdot}(X \wedge M \mathbb{Z} / 2)$ is $\Delta$-acyclic, then the argument used for $p$ odd still applies. Hence $K^{\cdot}\left(X \wedge M \mathbb{Z} / 2 \wedge C_{\eta}\right)$ is finitely-generated over $\Lambda$. But for any $Y$ there is a short exact sequence

$$
0 \longrightarrow K^{\prime} Y(1) \longrightarrow K^{\prime}\left(Y \wedge C_{\eta}\right) \longrightarrow K^{\prime} Y \longrightarrow 0
$$

since $\eta$ is zero in complex $K$-theory. Hence $K^{\cdot}(X \wedge M \mathbb{Z} / 2)$ is also finitely-generated over $\Lambda$, completing the proof of the theorem.

8.2. Functional and Brown-Comenetz duality. We say that $X$ is weakly $d u$ alizable if the natural map

$$
X \longrightarrow D^{2} X
$$

is an equivalence. Any dualizable object is weakly dualizable, but the converse is false as we will see.

The map $X \longrightarrow D^{2} X$ generalizes to a "double $Y$-duality" map

$$
\phi_{X, Y}: X \longrightarrow F(F(X, Y), Y)
$$

adjoint to the evaluation map $X \wedge F(X, Y) \longrightarrow Y$, which in turn is adjoint to the identity map of $F(X, Y)$.

Proposition 8.4. $X$ is weakly dualizable if and only if for every invertible object $W$, the double $W$-duality map $\phi_{X, W}: X \longrightarrow F(F(X, W), W)$ is an equivalence. More generally, if $\phi_{X, W}$ is an equivalence for one invertible $W$, it is an equivalence for all invertible $W$.

This follows easily from the fact ([13, A.2.8) that any invertible $W$ is dualizable, with $D W=W^{-1}$.

There is another kind of duality in $L_{K(1)} \mathcal{S}$, called Brown-Comenetz duality (see [12]). Fix $E$ and consider the functor

$$
X \mapsto\left(\pi_{0}(E \wedge X \wedge \mathcal{N})\right)^{\#} .
$$

This functor is cohomological and hence is representable. The representing spectrum $d E$ is the Brown-Comenetz dual of $E$, denoted $\hat{I} E$ in [12]. For example, Proposition 3.5 can be interpreted as saying:

Proposition 8.5. $d K \cong \Sigma K$.

It follows from the definition that we have a universal coefficient theorem.

Proposition 8.6. There is a natural short exact sequence

$$
0 \longrightarrow\left(t_{\mathbb{Z}_{p}} \pi_{-n} E\right)^{\#} \longrightarrow \pi_{n} d E \longrightarrow H o m\left(\pi_{1-n} E, \mathbb{Z}_{p}\right) \longrightarrow 0,
$$

where $t_{\mathbb{Z}_{p}}(-)$ denotes p-torsion. 
In particular, if $E$ has $p$-torsion homotopy groups, then the homotopy groups of the Brown-Comenetz dual are just the Pontrjagin duals of the homotopy groups of E.

As a formal consequence of the definitions, we have:

Proposition 8.7. There is a natural equivalence $d E \cong F\left(E, d S^{0}\right)$.

We next determine $d S^{0}$. When $p$ is odd, this is easy using Proposition 8.5 and Lemma 8.20 below. However, we will give a different proof here that makes the isomorphism of functors explicit.

Theorem 8.8. If $p$ is odd, $d S^{0} \cong S^{2}$.

If $p=2, d S \cong \Sigma^{2} W$, where $W$ is the invertible spectrum of Theorem 7.4 .

Proof. Suppose $p$ is odd. From the universal coefficient theorem we have the short exact sequence

$$
0 \longrightarrow \pi_{n+1} S^{2} \otimes \mathbb{Z} / p^{\infty} \longrightarrow \pi_{n} S^{2} \wedge \mathcal{N} \longrightarrow \pi_{n} S^{2}\left[p^{\infty}\right] \longrightarrow 0
$$

and hence

$$
\mathbb{Z} / p^{\infty}=\pi_{1} S^{2} \otimes \mathbb{Z} / p^{\infty} \stackrel{\cong}{\cong} \pi_{0} S^{2} \wedge \mathcal{N} .
$$

This yields a natural transformation of cohomology theories

$$
\zeta:\left[X, S^{2}\right] \longrightarrow\left(\pi_{0} X \wedge \mathcal{N}\right)^{\#}
$$

given by $\zeta(f)=\pi_{0} f \wedge \mathcal{N}$, and we will show $\zeta$ is an isomorphism.

By Theorem 4.3 and the periodicity of $K$, it is enough to show $\zeta$ is an isomorphism for $X=K, \Sigma K$. For $X=K$ both source and target are zero, so there is nothing to prove. For $X=\Sigma K, \zeta$ factors as

$$
\begin{gathered}
{\left[\Sigma K, S^{2}\right] \longrightarrow \operatorname{Hom}\left(\pi_{1} \Sigma K, \pi_{1} S^{2}\right) \longrightarrow \operatorname{Hom}\left(\pi_{1} \Sigma K \otimes \mathbb{Z} / p^{\infty}, \pi_{1} S^{2} \otimes \mathbb{Z} / p^{\infty}\right)} \\
\longrightarrow \operatorname{Hom}\left(\pi_{0} \Sigma K \wedge \mathcal{N}, \pi_{0} S^{2} \wedge \mathcal{N}\right),
\end{gathered}
$$

where the maps are the obvious ones. The first map is an isomorphism by direct calculation: $\left[\Sigma K, S^{2}\right]=\mathbb{Z}_{p}$, generated by $\Sigma \delta$, where $\delta: K \longrightarrow S^{1}$ is the coboundary map in the cofibre sequence $S^{0} \longrightarrow K \longrightarrow K$. The second map is just the evident isomorphism $\operatorname{Hom}\left(\mathbb{Z}_{p}, \mathbb{Z}_{p}\right) \cong \operatorname{Hom}\left(\mathbb{Z} / p^{\infty}, \mathbb{Z} / p^{\infty}\right)$. The third map is an isomorphism because $\pi_{0} \Sigma K \wedge \mathcal{N}=0=\pi_{S}^{2} \wedge \mathcal{N}$.

The proof for $p=2$ is almost identical, but with one additional twist. In the universal coefficient theorem we have

$$
0 \longrightarrow \pi_{1} \Sigma^{2} W \otimes \mathbb{Z} / 2^{\infty} \longrightarrow \pi_{0} \Sigma^{2} W \wedge \mathcal{N} \longrightarrow \pi_{0} \Sigma^{2} W\left[2^{\infty}\right] \longrightarrow 0,
$$

with $\pi_{1} \Sigma^{2} W \otimes \mathbb{Z} / 2^{\infty} \cong \mathbb{Z} / 2^{\infty}$ as before. On the other hand, $\pi_{0} \Sigma^{2} W=\mathbb{Z} / 2$, and hence the short exact sequence has the form

$$
0 \longrightarrow \mathbb{Z} / 2^{\infty} \longrightarrow \pi_{0} \Sigma^{2} W \wedge \mathcal{N} \longrightarrow \mathbb{Z} / 2 \longrightarrow 0 .
$$

This sequence splits, of course, but there are two choices of splitting and no apparent reason to prefer one over the other. Nevertheless, we simply choose a splitting $\alpha: \pi_{0} \Sigma^{2} W \wedge \mathcal{N} \longrightarrow \mathbb{Z} / 2^{\infty}$, yielding a natural transformation of cohomology theories

$$
\zeta:\left[X, \Sigma^{2} W\right] \longrightarrow \operatorname{Hom}\left(\pi_{0} X \wedge \mathcal{N}, \pi_{0} \Sigma^{2} W \wedge \mathcal{N}\right) \longrightarrow \operatorname{Hom}\left(\pi_{0} X \wedge \mathcal{N}, \mathbb{Z} / 2^{\infty}\right)
$$


Note that if $\pi_{0} X$ is torsion-free, the splitting $\alpha$ is irrelevant; we can regard $\zeta$ as a homomorphism

$$
\left[X, \Sigma^{2} W\right] \longrightarrow \operatorname{Hom}\left(\pi_{1} X \otimes \mathbb{Z} / 2^{\infty}, \pi_{1} \Sigma^{2} W \otimes \mathbb{Z} / 2^{\infty}\right) \cong \operatorname{Hom}\left(\mathbb{Z} / 2^{\infty}, \mathbb{Z} / 2^{\infty}\right) .
$$

Taking $X=K, \Sigma K$, the rest of the proof is identical to the odd primary case.

Corollary 8.9. $d S^{0}$ is invertible.

In fact one can prove the corollary a priori; see [12, Theorem 10.2e. The proof there simplifies dramatically in the case $n=1$.

Call $X$ weakly Brown-Comenetz dualizable if the double $d S^{0}$-duality map is an equivalence. Combining Theorem 8.8 with Proposition 8.4 we have:

Proposition 8.10. $X$ is weakly dualizable if and only if $X$ is weakly BrownComenetz dualizable.

The next theorem is the main result of this subsection.

Theorem 8.11. $X$ is weakly Brown-Comenetz dualizable if and only if $\pi_{n} X$ is finitely-generated over $\mathbb{Z}_{p}$ for all $n$.

Proof. By smashing with $M \mathbb{Z} / p$, we easily reduce to the following special case.

Lemma 8.12. Suppose $X$ has a finite exponent. Then $X$ is weakly Brown-Comenetz dualizable if and only if $X$ has finite homotopy groups.

This lemma has an algebraic antecedent.

Lemma 8.13. Let $A$ be an abelian p-torsion group. Then

(a) $A^{\#}$ is torsion if and only if $A$ has a finite exponent.

(b) The natural homomorphism $A \longrightarrow A^{\# \#}$ is an isomorphism if and only if $A$ is finite.

Now observe that by the universal coefficient theorem above, for a finite exponent

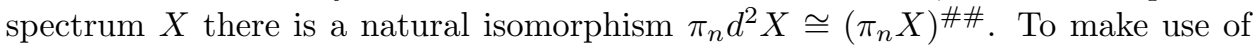
this we need to know further:

Lemma 8.14. Suppose $X$ has finite exponent. Then the map $\pi_{n} X \longrightarrow\left(\pi_{n} X\right)^{\# \# ~ i n-~}$ duced by $X \longrightarrow d^{2} X$ coincides with the natural homomorphism as in Lemma 8.13(b).

If the reader is willing to believe Lemma 8.14 then Lemma 8.12 follows Lemma 8.13, completing the proof of Theorem 8.11] However, the proof of Lemma 8.14 is not entirely trivial, so we will sketch it here.

For arbitrary $X, Y$, let $\epsilon_{X, Y}$ denote the natural homomorphism

$$
\pi_{0} F(X, Y) \longrightarrow \operatorname{Hom}\left(\pi_{0} X, \pi_{0} Y\right) .
$$

Then the following diagram commutes:

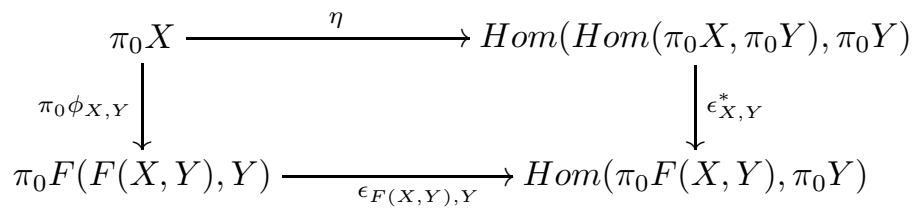

Here $\eta$ is the algebraic analogue of $\phi$. We cannot apply this directly to $Y=$ $d S^{0}$ because of the smash product with $\mathcal{N}$ in the definition of Brown-Comenetz 
duality. However, for any torsion spectrum $X$ and arbitrary spectrum $Y$ there is a commutative diagram

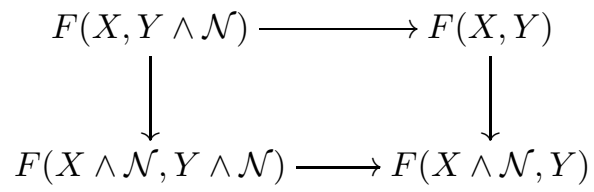

in which all four maps are homotopy equivalences. The maps are the natural ones arising from the canonical map $\mathcal{N} \longrightarrow S^{0}$. Furthermore, if $X$ has finite exponent, then so does $F(X, Y)$.

Now if $p$ is odd, taking $Y=d S^{0} \wedge \mathcal{N}$ in the first diagram we find that:

(i) $\eta$ is the Pontrjagin double duality map;

(ii) $\phi_{X, Y}$ is the Brown-Comenetz double duality map;

and

(iii) the two $\epsilon$ maps are Brown-Comenetz duality isomorphisms.

This completes the proof for $p$ odd.

When $p=2$ we extend the first diagram as follows, using the homomorphism $\alpha: \pi_{0}(d S \wedge \mathcal{N}) \longrightarrow \mathbb{Z} / 2^{\infty}$ defined earlier and writing $h$ in place of Hom to save space:

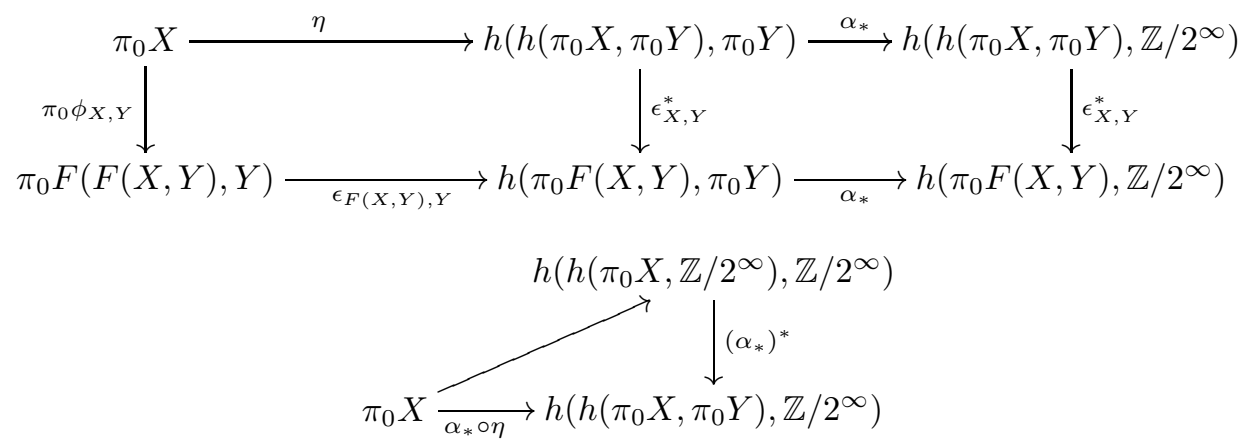

Here we have:

(i) the diagonal map is again the Pontrjagin double duality map;

(ii) $\phi_{X, Y}$ is again the Brown-Comenetz double duality map;

(iii) the composite map in the bottom row of the first diagram is an isomorphism as in the odd primary case;

(iv) the vertical composite $\epsilon_{X, Y}^{*} \circ\left(\alpha_{*}\right)^{*}$ is an isomorphism as in the odd primary case.

The 2-primary case of Lemma 8.14 now follows as before.

We conclude with an important example for $p=2$.

\section{Proposition 8.15.}

$$
d K O \cong \Sigma^{-3} K O .
$$

Proof. We first show:

Lemma 8.16. $D K O \cong \Sigma^{-1} K O$.

Consider the fibre sequence

$$
\Sigma^{-1} K O \stackrel{\Sigma^{-1} T}{\longrightarrow} \Sigma^{-1} K O \stackrel{\delta}{\longrightarrow} S^{0} .
$$


Let $\phi: \Sigma^{-1} K O \longrightarrow D K O=\mathcal{F}\left(K O, S^{0}\right)$ denote the composite

$$
\Sigma^{-1} K O \stackrel{\tau}{\longrightarrow} \mathcal{F}\left(K O, \Sigma^{-1} K O\right) \stackrel{\delta_{*}}{\longrightarrow} \mathcal{F}\left(K O, S^{0}\right),
$$

where $\tau$ is adjoint to the desuspended ring multiplication. A direct check on homotopy groups shows that $\phi$ is a weak equivalence, proving the lemma.

We then have

$$
\begin{aligned}
d K O \cong \mathcal{F}\left(K O, \Sigma^{2} W\right) \cong \mathcal{F}\left(K O, S^{0}\right) \wedge \Sigma^{2} W & \cong \Sigma^{-1} K O \wedge \Sigma^{2} W \\
& \cong \Sigma^{-1} K O \wedge S^{-2}
\end{aligned}
$$

where the four equivalences follow respectively from Theorem 8.8 , the fact that $W$ is invertible and hence dualizable, the lemma, and Proposition 7.5.

8.3. Compact topologies on $[-, X]$. The natural topology on $[X, Y]([13, \S 4.4)$ is the topology with neighborhood base at the origin given by the kernels of all maps $f^{*}:[X, Y] \longrightarrow[W, Y]$, where $(W, f)$ ranges over all maps $f$ of small objects $W$ to $X$.

Proposition 8.17. The natural topology on $[W, X]$ is compact Hausdorff for all $W$ if and only if $\pi_{n} X$ is finitely-generated over $\mathbb{Z}_{p}$ for all $n$.

Proof. Suppose $[Y, X]$ is compact Hausdorff in the natural topology. If $Y$ is small, then $[Y, X]$ is also discrete, and hence finite. Taking $Y=M \mathbb{Z} / p$, it follows that $X$ has finite $\bmod p$ homotopy groups and hence $\pi_{n} X$ is finitely-generated over $\mathbb{Z}_{p}$ for all $n$.

Conversely, suppose $\pi_{n} X$ is finitely-generated over $\mathbb{Z}_{p}$ for all $n$. By Theorem 8.11, $X$ is weakly Brown-Comenetz dualizable and hence $X=d Y$ where $Y$ also has homotopy groups finitely-generated over $\mathbb{Z}_{p}$.

Now observe that for any $E$, the functor represented by $d E$ takes values in the category of compact Hausdorff $\mathbb{Z}_{p}$-modules and continuous homomorphisms, since it is Pontrjagin dual to a functor taking values in discrete $p$-torsion groups. Hence it will suffice to show that the natural topology agrees with this Brown-Comenetz topology.

By Theorem 6.1 an object is small if and only if it is $M \mathbb{Z} / p$-finite. Hence $\pi_{n}(W \wedge Y)$ is finite for all small $W$ and all $n$. It follows easily that the two topologies agree, as desired.

More generally one can ask whether there is any lift of $[-, X]$ to compact $\mathbb{Z}_{p^{-}}$ modules.

Proposition 8.18. The functor represented by $X$ lifts to the category of compact $\mathbb{Z}_{p}$-modules and continuous homomorphisms if and only if $X$ is in the image of the Brown-Comenetz duality functor.

Proof. If $X=d Y$ for some $Y$, then $[-, X]$ lifts to compact Hausdorff $\mathbb{Z}_{p}$-modules, as noted in the proof of the preceeding proposition.

Conversely, suppose $[-, X]$ lifts to a functor $\mathfrak{h}$ to compact Hausdorff $\mathbb{Z}_{p}$-modules. Then the Pontrjagin dual $\mathfrak{h}^{\#}$ is a homology functor and so is representable by Theorem 4.8: $(\mathfrak{h}(Z))^{\#}=\pi_{0} E \wedge Z \wedge \mathcal{N}$ for some $E$. Hence

$$
[Z, X]=\mathfrak{h}(Z)=[Z, d E]
$$

as required. 
8.4. $K$-theory of the dual as a module over the Iwasawa algebra. Let $X$ be a $K$-finite spectrum. In this section we obtain a universal coefficient formula for the $K$-theory of the Brown-Comenetz dual, as modules over $\Lambda^{\prime}$.

For any pro-group ring $\mathbb{Z}_{p}[[G]]$, we let $\chi$ denote the canonical anti-automorphism given by $\chi(g)=g^{-1}$. If $M$ is a left $\mathbb{Z}_{p}[[G]]$-module, let $M^{\chi}$ denote the right module defined by setting $x \alpha=\chi(\alpha) x$ for $\alpha \in \mathbb{Z}_{p}[[G]], x \in M$. Similarly, $\chi$ converts right modules to left modules.

When $G=\Gamma^{\prime}$, the fact that $\Gamma^{\prime}$ is commutative confuses the issue. To help clarify the discussion below, we will sometimes speak of left versus right actions as though $\Gamma^{\prime}$ were noncommutative.

Theorem 8.19. Let $X \in L_{K(1)} \mathcal{S}$ be $K$-finite. Then there is a spectral sequence of $\Lambda^{\prime}$-modules with

$$
E_{2}^{s, t}=\operatorname{Ext}_{\Lambda^{\prime}}^{s}\left(K^{\epsilon} X, \Lambda^{\prime}\right)^{\chi}(m),
$$

where $\epsilon=0,1$ and $t=2 m+\epsilon$, converging to $K^{1-(t-s)} d X$.

Remark. To clarify the $\Lambda^{\prime}$-module structure on the $E_{2}$-term, recall that since $\Lambda^{\prime}$ is commutative, for any $M, N$ there is a natural module structure on $\operatorname{Ext}_{\Lambda^{\prime}}(M, N)$. The module structure on the $E_{2}$-term in the theorem is obtained from this one by applying the functor $\chi$ and then taking the $m$-fold Tate twist.

Proof. Since $X$ is weakly Brown-Comenetz dualizable, we have

$$
\left[d X, \Sigma^{n} K\right] \cong\left[d\left(\Sigma^{n} K\right), d^{2} X\right] \cong\left[\Sigma^{1-n} K, X\right] .
$$

This an isomorphism of left $\Lambda^{\prime}$-modules, where $\Lambda^{\prime}$ acts on $\left[\Sigma^{1-n} K, X\right]$ by

$$
\lambda \cdot\left(f: \Sigma^{-n}(\Sigma K) \longrightarrow X\right)=f \circ\left(\Sigma^{-n} d \lambda\right) .
$$

Lemma 8.20. $d \lambda=\Sigma \chi(\lambda)$.

Proof. There is a natural isomorphism

$$
\pi_{2 n+1} d K \cong \operatorname{Hom}_{\mathbb{Z}_{p}}\left(\pi_{-2 n} K, \mathbb{Z}_{p}\right)=H_{o m} \mathbb{Z}_{p}\left(\mathbb{Z}_{p}(-n), \mathbb{Z}_{p}\right) .
$$

Identifying $\pi_{2 n+1} d K$ with $\pi_{2 n} K$, we see that the action of $d \lambda$ on $\pi_{2 n} K$ is the same as the action of $\chi(\lambda)$. Since the representation of $\Lambda^{\prime}$ on $\pi_{*} K$ is faithful, this proves the lemma.

Now consider the Adams spectral sequence converging to $\left[\Sigma^{n} K, X\right]$. This is a spectral sequence of left $\Lambda^{\prime}$-modules, where

$$
\lambda \cdot\left(f: \Sigma^{n} K \longrightarrow X\right)=f \circ \Sigma^{n} \chi(\lambda) .
$$

By the lemma, on the abutment this module structure corresponds to the usual module structure on $K^{1-n} d X$. So it remains to determine the module structure on the $E_{2}$-term. Taking $t=2 m$ to illustrate, we have

$$
E_{2}^{s, t}=\operatorname{Ext}_{\Lambda^{\prime}}^{s}\left(K^{0} X, K^{0} \Sigma^{2 m} K\right) .
$$

In general, the Ext has a $\Lambda^{\prime}$-module structure only because $\Lambda^{\prime}$ happens to be commutative. Call this the "standard" structure, and note that it can be computed by letting $\Lambda^{\prime}$ act by post-composition on $\left[\Sigma^{2 m} K, K\right]$. The structure we want, however, is obtained by precomposition with $\Sigma^{2 m} \chi(\lambda)$. Using the relation

$$
\gamma \beta^{m}=c(\gamma)^{m} \beta^{m} \circ \Sigma^{2 m} \gamma,
$$


where $\gamma \in \Gamma^{\prime}$ and $\beta$ is the Bott class, we see that this module structure is obtained from the standard one by applying $\chi$ and then the $m$-fold Tate twist. This completes the proof of the theorem.

Example. Take $p$ odd and suppose $K \cdot X$ is $\Lambda$-torsion with no finite submodules (so that $K^{\cdot} X$ has projective dimension one as a $\Lambda$-module). To simplify the discussion, assume also that $K^{1} X=0$. Then $K^{1} d X=0$ and

$$
K^{0} d X=\operatorname{Ext}_{\Lambda^{\prime}}^{1}\left(K^{0} X, \Lambda^{\prime}\right)^{\chi}(1) .
$$

For instance, if $X=S^{2 n}$ we get

$$
\operatorname{Ext}_{\Lambda^{\prime}}^{1}\left(\mathbb{Z}_{p}(n), \Lambda^{\prime}\right)^{\chi}(1)=\mathbb{Z}_{p}(n)^{\chi}(1)=\mathbb{Z}_{p}(1-n),
$$

confirming that $d S^{2 n}=S^{2-2 n}$.

See Proposition 10.10 for a description of $K \cdot d X$ up to pseudo-isomorphism.

\section{Pseudo-equivalence of $K(1)$-Local spectra}

In this section we assume $p$ is odd. The $K$-local, $p$-local spectra were classified up to equivalence by Bousfield [3]. The classification obtained here is much coarser, but is nonetheless enlightening, and useful in applications such as [16. We also note that according to unpublished work of Frenke [7, for $p$ odd the category of $K$-local spectra is equivalent to a certain category of chain complexes. We will not make any use of this.

We begin by observing (cf. [3]).

Proposition 9.1. Suppose $X \in L_{K(1)} \mathcal{S}$. Then $X$ is uniquely determined by the $\Lambda^{\prime}$-module $K^{\cdot} X$ under either of the following hypotheses:

a) One of $K^{0} X, K^{1} X$ vanishes,

b) $K^{\cdot} X$ has projective dimension at most one as a $\Lambda^{\prime}$-module.

Proof. The obstruction to realizing an isomorphism $\phi: K \cdot X \cong K^{\prime} Y$ by a map $Y \longrightarrow X$ is the element

$$
d_{2} \phi \in \operatorname{Ext}_{\Lambda^{\prime}}^{2}\left(K^{\cdot} X, K^{\cdot} \Sigma Y\right)
$$

The $E x t^{2}$ vanishes for parity reasons in case a), and by assumption in case b); in fact, in case b) $X$ splits as a wedge of two objects satisfying condition a).

Let $N^{\cdot}=N^{0} \oplus N^{1}$ be a $\Lambda^{\prime}$-module. Following [3] we then define the generalized Moore spectrum $\mathcal{M}_{N}$. as follows: if one of $N^{0}, N^{1}$ is zero, $\mathcal{M}_{N}$. is the unique object with $K \cdot \mathcal{M}_{N}=N^{*}$. In general we set $\mathcal{M}_{N}=\mathcal{M}_{N_{0}} \bigvee \mathcal{M}_{N_{1}}$.

In many cases we are given only a $\Lambda$-module $M$. We then write $(M, i)$ for the corresponding $\Lambda^{\prime}$-module with $\Delta$ acting as $\omega^{i}$. If $i$ is omitted it is to be taken as zero. As examples of this notation we have

$$
\mathcal{M}_{\Lambda, i}=e_{i} K, \quad M_{\Lambda}=e_{0} K=E(1) .
$$

A morphism $X \longrightarrow Y$ is a pseudo-equivalence if it has small cofibre. From Theorem 6.1 we have at once:

Proposition 9.2. $f: X \longrightarrow Y$ is a pseudo-equivalence if and only if $K \cdot f$ is a pseudo-isomorphism. 
Now call an object $Y$ elementary if $K \cdot Y$ is an elementary $\Lambda$-module. Equivalently, by Proposition 9.1, $Y$ is a finite coproduct of spectra of the form $\Sigma^{r} \mathcal{M}_{\Lambda / q^{k}, i}$, where $r=0,1, q$ is either $0, p$, or an irreducible distinguished polynomial, and $0 \leq i \leq p-2$.

Theorem 9.3. Every $K$-finite spectrum $X$ is pseudo-equivalent to an elementary spectrum $\mathcal{M}_{E}$, unique up to equivalence. The pseudo-equivalence can be taken of the form

$$
X \stackrel{\sim}{\longrightarrow} Y \stackrel{\sim}{\longleftarrow} \mathcal{M}_{E}
$$

for some intermediate spectrum $Y$.

Lemma 9.4. Let $M$ be a finitely-generated $\Lambda$-module. Then there is a torsion-free submodule $N$ such that $t M \oplus N$ has finite index in $M$.

Proof. Suppose first that $t M$ is finite, and consider the filtration $\mathfrak{m}^{k} M \supseteq \mathfrak{m}^{k+1} M \supseteq$ .... Then $\bigcap_{k} \mathfrak{m}^{k} M=0$ and hence $\bigcap_{k}\left(\mathfrak{m}^{k} M\right) \cap t M=0$. Since $t M$ is finite, this forces $\left(\mathfrak{m}^{k} M\right) \cap t M=0$ for $k>>0$. Then we can take $N=\mathfrak{m}^{k} M, k>>0$.

In the general case there is a pseudo-retraction $\phi: M \longrightarrow t M$; i.e., a homomorphism $\phi$ whose restriction to $t M$ is a pseudo-isomorphism. (This follows from the proof of the classification theorem.) Then the kernel $L$ of $\phi$ has finite torsion submodule, and hence has a torsion-free submodule $N$ of finite index as shown above. It follows that $t M \oplus N$ has finite index, as desired.

Corollary 9.5. There is a pseudo-isomorphism $L \oplus N \stackrel{\sim}{\longrightarrow} M$ with $L$ elementary torsion and $N$ torsion-free.

Proof of the theorem. Take $M=K \cdot X$, choose a pseudo-isomorphism $j: L \oplus$ $N \longrightarrow M$ as in the corollary and let $Y=\mathcal{M}_{L \oplus N}$. Then choose a pseudo-isomorphism $k: L \oplus N \longrightarrow E$ with $E$ elementary. Since $L \oplus N$ has projective dimension at most one, the diagram of $\Lambda^{\prime}$-modules

$$
K X \stackrel{\sim}{\longleftarrow} L \oplus N \stackrel{\sim}{\longrightarrow} E
$$

can be realized by a diagram of spectra

$$
X \stackrel{\sim}{\longrightarrow} Y \stackrel{\sim}{\longleftarrow} \mathcal{M}_{E}
$$

as desired.

In general, there is no pseudo-equivalence $X \longrightarrow \mathcal{M}_{E}$ or $\mathcal{M}_{E} \longrightarrow X$.

Example. Let $X=\mathcal{M}_{\mathfrak{m}}$, so $E=\Lambda$. There is no pseudo-isomorphism $\Lambda \longrightarrow \mathfrak{m}$, and hence there is no pseudo-equivalence $X \longrightarrow \mathcal{M}_{E}$.

Example. An easy calculation shows that

$$
\left[\Sigma^{-1} \mathcal{M}_{\Lambda}, M \mathbb{Z} / p\right]=\operatorname{Ext}_{\Lambda^{\prime}}^{2}(\mathbb{Z} / p, \Lambda)=\mathbb{Z} / p .
$$

Let $\alpha: \Sigma^{-1} \mathcal{M}_{\Lambda} \longrightarrow M \mathbb{Z} / p$ be a generator, and let let $X$ be the cofibre. Then $E=\Lambda$, and we claim that there is no pseudo-equivalence $\mathcal{M}_{\Lambda} \longrightarrow X$. To see this, note that $K^{0} X=\Lambda$ and $K^{1} X=\mathbb{Z} / p$. Since any pseudo-isomorphism of free $\Lambda$-modules is an isomorphism by Corollary 2.5, a pseudo-equivalence $\mathcal{M}_{\Lambda} \longrightarrow X$ would induce an isomorphism on $K^{0}$. Hence $X$ would split as $M \mathbb{Z} / p \bigvee \mathcal{M}_{\Lambda}$, contradicting the fact that $\alpha$ is essential. 
Taking the wedge of the preceeding two examples gives an example with no pseudo-equivalence in either direction; presumably one could also find an indecomposable example.

In the torsion case, however, we can get maps going both ways.

Theorem 9.6. Suppose $X$ is $K$-finite and $K X$ is $\Lambda$-torsion, with associated elementary module $E^{*}$. Then there are pseudo-equivalences $\mathcal{M}_{E} \longrightarrow X$ and $X \longrightarrow \mathcal{M}_{E}$.

To prove this we need a lemma of independent interest. Consider arbitrary $X, Y \in L_{K(1)} \mathcal{S}$ and the Adams spectral sequence converging to $[X, Y]$. Since the $E_{2}$-term is canonically identified with certain Ext-groups over $\Lambda^{\prime}$, it has a $\Lambda^{\prime}$-module structure.

Lemma 9.7. $d_{2}: E_{2}^{0, t} \longrightarrow E_{2}^{2, t+1}$ is a homomorphism of $\Lambda^{\prime}$-modules.

Proof. There is a formula for $d_{2}$ due to Bousfield [3], which in our context reads as follows: if $f \in E_{2}^{0, t}$, then

$$
d_{2} f=\kappa_{Y} f+(-1)^{t+1} f \kappa_{X} .
$$

Here the invariant $\kappa_{X}$ lies in

$$
E_{2}^{2,1}(X, X)=\operatorname{Ext}_{\Lambda^{\prime}}^{2}\left(K^{\cdot} X, K^{\cdot} \Sigma X\right)
$$

It is the obstruction to an equivalence of $X$ with the generalized Moore spectrum associated to $K \cdot X$. The products with $f$ are Yoneda products. Taking $t=0$ for convenience, we have

$$
\begin{gathered}
d_{2}(\lambda f)=\kappa_{Y}(\lambda f)-(\lambda f) \kappa_{X}, \\
\lambda d_{2} f=\lambda\left(\kappa_{Y} f\right)-\lambda\left(f \kappa_{X}\right) .
\end{gathered}
$$

Since the Yoneda product is associative, we have only to show that

$$
\lambda \kappa_{Y}=\kappa_{Y} \lambda \in \operatorname{Ext}_{\Lambda^{\prime}}^{2}\left(K^{\cdot} Y, K^{\cdot} Y\right) .
$$

But this is true because for any commutative ring $R$, the $R$-module structure on $\operatorname{Ext}_{R}^{*}(M, N)$ can be computed in either variable.

To prove the theorem, first recall that since $K \cdot X$ is $\Lambda$-torsion, there are pseudoisomorphisms $\alpha: E \cdot K^{\cdot} X$ and $\beta: K^{\cdot} X \longrightarrow E^{\cdot}$. Since $E$ has projective dimension at most one, we can realize $\alpha$ by a pseudo-equivalence $X \longrightarrow \mathcal{M}_{E}$.

Now consider $\beta \in E_{2}^{0,0}\left(\mathcal{M}_{E}, X\right)$. Let $\lambda \in \Lambda$ be any irreducible element not in the support of $K^{\cdot} X$. Then for all $k, \lambda^{k} \beta$ is still a pseudo-isomorphism. But $E_{2}^{2,1}$ is an $E x t^{2}$ of two finitely-generated modules and therefore is finite. Hence for $k>>0$, $\lambda^{k} E_{2}^{2,1}=0$. For $k>>0$ it then follows from the lemma that $d_{2}\left(\lambda^{k} \beta\right)=\lambda^{k} d_{2} \beta=0$. Hence $\lambda^{k} \beta$ can be realized by a pseudo-equivalence $\mathcal{M}_{E} \longrightarrow X$.

\section{ThiCK SUBCATEgORIES OF $K$-Finite SPECTRA}

The goal of this section is to classify and study the thick subcategories of the $K$-finite spectra $\mathcal{C}$. We continue to assume $p$ is odd.

We call a set of primes $S \subset$ Spec $\Lambda^{\prime}$ fit if it is closed under specialization and Tate twisting. Given a fit subset $S$, let $\mathcal{C}_{S}$ denote the full subcategory of $\mathcal{C}$ whose objects $X$ have $\operatorname{Supp} K^{*} X \subset S$.

Proposition 10.1. If $S$ is a fit subset of $S p e c \Lambda^{\prime}$, then $\mathcal{C}_{S}$ is a thick subcategory. 
Proof. Since $S$ is closed under Tate twisting, $\mathcal{C}_{S}$ is closed under suspension. Standard arguments then show that $\mathcal{C}_{S}$ is thick.

The main result of this section is the converse.

Theorem 10.2. Let $\mathcal{C}$ be a thick subcategory of the $K$-finite spectra $\langle K\rangle$. Then there is a unique fit set of primes $S$ such that $\mathcal{C}=\mathcal{C}_{S}$.

We assume from now on that $\mathcal{C}$ is a nontrivial thick subcategory.

Lemma 10.3. $\mathcal{C}$ contains every small object $F$.

Proof. By Theorem 6.3, it suffices to show $\mathcal{C}$ contains one nontrivial small object.

By assumption $\mathcal{C}$ contains a nontrivial object $X$. Smashing with $M \mathbb{Z} / p$, we can assume $K^{\cdot} X$ is $\Lambda$-torsion. Let $\lambda \in \Lambda$ be any nonunit whose prime factors are disjoint from the support of $K^{\cdot} X$. Then multiplication by $\lambda$ is a pseudoisomorphism of $K^{\cdot} X$. Furthermore, by Lemma 9.7, there is a self-map $f: X \longrightarrow X$ realizing multiplication by $\lambda$. The cofibre of $f$ is nontrivial, is in $\mathcal{C}$ and has finite $K$. Hence $C_{f}$ is small by Theorem 6.1, proving the lemma.

Corollary 10.4. $\mathcal{C}$ is closed under pseudo-equivalence.

Lemma 10.5. Suppose $X \in \mathcal{C}$ and $Y$ is $K$-finite with Supp $K^{\cdot} Y \subset \operatorname{Supp} K^{\cdot} X$. Then $Y \in \mathcal{C}$.

Proof. By Corollary 10.4 and Theorem 9.3 . we may assume that $X$ and $Y$ are elementary spectra. If $K X$ is not $\Lambda$-torsion, it follows that $\mathcal{M}_{\Lambda} \in \mathcal{C}$; hence $\mathcal{C}$ consists of all $K$-finite spectra and we are done. Hence we may assume $K \cdot X$ is $\Lambda$ torsion. Since $\mathcal{C}$ is closed under retracts, we reduce further to the case $X=\mathcal{M}_{\Lambda / q^{i}}$, $Y=\mathcal{M}_{\Lambda / q^{j}}$ for some irreducible element $q$.

Let $f: X \longrightarrow X$ be a self-map realizing multiplication by $q$. The cofibre $Z$ satisfies $K^{0} Z=\Lambda / q=K^{1} Z$ and hence by Proposition 9.1b),

$$
Z \cong \mathcal{M}_{\Lambda / q} \bigvee \Sigma \mathcal{M}_{\Lambda / q}
$$

Hence $\mathcal{M}_{\Lambda / q} \in \mathcal{C}$. It then follows by induction on $j$ that $Y \in \mathcal{C}$, completing the proof of the lemma.

Theorem 10.2 follows immediately from the lemma: let $S$ denote the union of the supports of all $K^{n} X$ for all $X \in \mathcal{C}$ and all $n \in \mathbb{Z}$. Then $S$ is fit and $\mathcal{C}=\mathcal{C}_{S}$.

10.1. Examples. If $S$ is a set of primes that is not necessarily fit, it will be convenient to let $\mathcal{C}_{S}$ denote the thick subcategory corresponding to the fit subset generated by $S$.

1. Let $\mathcal{C}$ be the thick subcategory of small objects. Then $\mathcal{C}=C_{\{\mathfrak{m}\}}$ by Theo$\operatorname{rem} 6.1$

2. Let $\mathcal{C}$ be the thick subcategory of dualizable objects. Then $\mathcal{C}=\mathcal{C}_{S}$, where $S$ is the set of irreducible distinguished polynomials. This is clear from Theorem 5.1.

3. Let $\mathcal{C}$ be the thick subcategory of finite exponent objects. Then $\mathcal{C}=\mathcal{C}_{\{p\}}$. (Note that $p$ means $p \Lambda$.)

4. Let $\mathcal{C}$ be the thick subcategory of objects with all homotopy groups finite. Then $\mathcal{C}=\mathcal{C}_{S}$, where $S$ is the complement of the set of Tate primes in the set of all height one primes.

To see this, note that by Proposition 2.3, in the Adams spectral sequence for $\pi_{*} X$, each $E_{\infty}^{0, *}$ has finite index in $E_{2}^{0, *}$. Hence $E_{\infty}^{0, *}$ is finite in each degree if and 
only if $\operatorname{Hom}_{\Lambda^{\prime}}\left(K \cdot X, \mathbb{Z}_{p}(n)\right)$ is finite for all $n$. By the structure theory of $\Lambda$-modules, these latter groups are finite if and only if $K^{\prime} X$ is $\Lambda$-torsion with support disjoint from the annihilator of the $\mathbb{Z}_{p}(n)$, i.e., disjoint from the set of Tate primes. In this case the groups $\operatorname{Ext}_{\Lambda^{\prime}}^{1}\left(K^{\prime} X, \mathbb{Z}_{p}(n)\right)$ are also finite. Since $\operatorname{Ext}_{\Lambda^{\prime}}^{2}(M, N)$ is finite for all finitely-generated modules $M, N$, it follows that $\mathcal{C}=\mathcal{C}_{S}$ as claimed.

5 . Let $\mathcal{C}$ be the thick subcategory of objects with almost all homotopy groups finite. Then $\mathcal{C}=\mathcal{C}_{S}$, where $S$ is the set of all height one primes.

This is similar to the preceding example. The support of $K X$ lies in the height one primes if and only if $K^{\cdot} X$ is a $\Lambda$-torsion module. Now if $K \cdot X$ is a $\Lambda$-torsion module, then since only finitely many Tate primes can occur in $\operatorname{Supp} K X$, only finitely many of the groups $\operatorname{Hom}_{\Lambda^{\prime}}\left(K^{\cdot} X, \mathbb{Z}_{p}(n)\right), \operatorname{Ext}_{\Lambda^{\prime}}^{1}\left(K \cdot X, \mathbb{Z}_{p}(n)\right)$ can be nonzero and $\mathbb{Z}_{p}$-torsion-free. Conversely if $K \cdot X$ is not a $\Lambda$-torsion module, then infinitely many of the groups $\operatorname{Hom}_{\Lambda^{\prime}}\left(K \cdot X, \mathbb{Z}_{p}(n)\right)$ are nonzero and $\mathbb{Z}_{p^{-}}$-torsion-free.

6. Let $\mathcal{C}$ be the thick subcategory generated by $L_{K(1)} S^{0}$. Then $\mathcal{C}=\mathcal{C}_{S}$, where $S$ is the set of Tate primes.

7. Let $\mathcal{C}$ be the thick subcategory generated by the invertible spectra. Then $\mathcal{C}=\mathcal{C}_{S}$, where $S$ is the set of linear distinguished polynomials.

10.2. Localization of finite spectra. We have seen that the localizations of finite $p$-torsion spectra form a thick subcategory and that this subcategory coincides with the small objects and with the $M \mathbb{Z} / p$-finite objects. There is no analogous statement for localizations of finite spectra. However, by Proposition 6.2 we have:

Lemma 10.6. The collection of all objects of the form $L_{K(1)} F, F$ finite, is closed under pseudo-equivalence.

Then we have:

Theorem 10.7. The following are equivalent:

(a) $X \cong L_{K(1)} F$ for some finite spectrum $F$.

(b) $K \cdot X$ has support in the Tate primes, and each Tate prime occurs with exponent at most one.

(c) $X$ is pseudo-equivalent to a wedge of spheres.

Proof. (a) $\Rightarrow($ b): We may assume that $F$ is the suspension spectrum of an actual finite complex. Using induction on the dimension of $F$, one easily proves the more precise statement:

Lemma 10.8. If $\operatorname{dim} F=2 d$, then $\operatorname{Supp} K^{0} X \subset\left\{\tau_{0}, \ldots, \tau_{d}\right\}$ and $\operatorname{Supp} K^{1} X \subset$ $\left\{\tau_{0}, \ldots, \tau_{d-1}\right\}$.

If $\operatorname{dim} F=2 d+1$, then Supp $K^{i} X \subset\left\{\tau_{0}, \ldots, \tau_{d}\right\}$ for $i=0,1$.

Furthermore, in all cases each $\tau_{i}$ that occurs has exponent one.

This yields the desired result.

(b) $\Rightarrow$ (c): By Theorem 9.3, $X$ is pseudo-equivalent to an elementary spectrum $\mathcal{M}_{E}$, where $E$ is a direct sum of $\mathbb{Z}_{p}(n)$ 's. In other words, $\mathcal{M}_{E}$ is a wedge of spheres.

(c) $\Rightarrow$ (a): This follows from Lemma 10.6 .

10.3. Duality on the thick subcategories. Let $\mathcal{C}$ be a thick subcategory of $L_{K(1)}^{f i n} \mathcal{S}$. Since $d:[X, Y] \longrightarrow[d Y, d X]$ is an isomorphism for $X, Y \in L_{K(1)}^{f i n} \mathcal{S}$, the image of $\mathcal{C}$ under $d$ is again a thick subcategory. Thus $d$ permutes the thick subcategories of $L_{K(1)}^{f i n} \mathcal{S}$, and so induces a permutation of the fit subsets of $\operatorname{Spec} \Lambda^{\prime}$. 
Proposition 10.9. Let $S$ be a fit subset of Spec $\Lambda^{\prime}$. Then $d \mathcal{C}_{S}=\mathcal{C}_{\chi(S)}$.

Here we recall that $\chi$ is the canonical anti-automorphism of $\Lambda^{\prime}$, regarding the latter as a pro-group ring. The proposition follows from a more precise description of the pseudo-isomorphism-type of $K^{\cdot} d X$. For an elementary $\Lambda^{\prime}$-module $E$, let

$$
E=t E \oplus f E
$$

denote the decomposition of $E$ into a $\Lambda$-torsion submodule $E$ and a $\Lambda$-free module $f E$. Let $E_{i}$ denote the elementary module associated to $K^{i} X$.

Proposition 10.10. There are pseudo-isomorphisms

$$
\begin{gathered}
K^{0} d X \sim t E_{0}^{\chi}(1) \oplus f E_{1}^{\chi}, \\
K^{1} d X \sim t E_{1}^{\chi} \oplus f E_{0}^{\chi} .
\end{gathered}
$$

Proof. One could start by replacing $X$ by a pseudo-equivalent elementary spectrum. It is also enlightening, however, to work directly with the spectral sequence of Theorem 8.19] To simplify notation we give the proof for the case of $K^{0}$.

For any $X, Y \in L_{K(1)}^{f i n} \mathcal{S}$, the second Adams filtration $\mathcal{A}^{2}=\mathcal{A}^{2}[X, Y]$ is finite. Hence the quotient map $[Y, X] \longrightarrow[Y, X] / \mathcal{A}^{2}$ is a pseudo-isomorphism of $\mathbb{Z}_{p^{-}}$ modules. (This step works even for $p=2$.)

Take $Y=\Sigma K$ and apply Theorem 8.19 to get a pseudo-isomorphism of $\Lambda^{\prime}$ modules

$$
K^{0} d X=[\Sigma K, X] \stackrel{\sim}{\longrightarrow}[\Sigma K, X] / \mathcal{A}^{2} .
$$

Now the short exact sequence

$$
0 \longrightarrow \mathcal{A}^{1} / \mathcal{A}^{2} \longrightarrow \mathcal{A}^{0} / \mathcal{A}^{2} \longrightarrow \mathcal{A}^{0} / \mathcal{A}^{1} \longrightarrow 0
$$

has $\mathcal{A}^{1} / \mathcal{A}^{2} \Lambda$-torsion (since $\operatorname{Ext}{ }_{\Lambda}^{1}(M, \Lambda)$ is always $\Lambda$-torsion), and $\mathcal{A}^{0} / \mathcal{A}^{1} \Lambda$-torsionfree (since $\operatorname{Hom}_{\Lambda}(M, \Lambda)$ is always $\Lambda$-free). It follows that the short exact sequence pseudo-splits as $\Lambda^{\prime}$-modules, so that there is a pseudo-isomorphism

$$
\mathcal{A}^{0} / \mathcal{A}^{2} \sim \mathcal{A}^{1} / \mathcal{A}^{2} \oplus \mathcal{A}^{0} / \mathcal{A}^{1}
$$

(This is false for $p=2$. There is a pseudo-splitting as $\Lambda$-modules, but not necessarily as $\Lambda^{\prime}$-modules.)

Whenever $X, Y \in L_{K(1)}^{f i n} \mathcal{S}$, the term $E_{2}^{s, t}$ of the Adams spectral sequence for $[Y, X]$ is finite for $s \geq 2$. Hence

$$
\mathcal{A}^{1} / \mathcal{A}^{2} \oplus \mathcal{A}^{0} / \mathcal{A}^{1} \sim E_{2}^{1,2} \oplus E_{2}^{0,1} .
$$

Then

$$
E_{2}^{0,1}=\operatorname{Hom}_{\Lambda^{\prime}}\left(K^{1} X, \Lambda^{\prime}\right)^{\chi} \sim \operatorname{Hom}_{\Lambda^{\prime}}\left(f E_{1}, \Lambda^{\prime}\right)^{\chi} \sim f E_{1}^{\chi}
$$

and

$$
E_{2}^{1,2}=\operatorname{Ext}_{\Lambda^{\prime}}^{1}\left(K^{0} X, \Lambda^{\prime}\right)^{\chi}(1) \sim \operatorname{Ext}_{\Lambda^{\prime}}^{1}\left(t E_{0}, \Lambda^{\prime}\right)^{\chi}(1)=t E_{0}^{\chi}(1) .
$$

At the last step we used the fact that if $R$ is any commutative ring and $x \in R$ is not a zero divisor, then $\operatorname{Ext}_{R}^{1}(R / x, R) \cong R / x$ as $R$-modules.

This completes the proof of Proposition 10.10, Note that Proposition 10.9 is an immediate consequence. 
10.4. Semi-discrete primes. Let $\Gamma_{n} \subset \Gamma$ denote the closed subgroup of index $p^{n}$. Thus $\Gamma / \Gamma_{n}$ is a finite cyclic group of order $p^{n}$, and in particular is discrete. Note that every homomorphism from $\Gamma$ to a discrete group factors through some $\Gamma / \Gamma_{n}$.

The group homomorphisms $\Gamma \longrightarrow \Gamma / \Gamma_{n}$ induce $\mathbb{Z}_{p}$-algebra homomorphisms $\mathbb{Z}_{p}[[\Gamma]] \longrightarrow \mathbb{Z}_{p}\left[\Gamma / \Gamma_{n}\right]$, or equivalently

$$
\rho_{n}: \Lambda \longrightarrow \Lambda / \omega_{n}
$$

where $\omega_{n}=(1+T)^{p^{n}}-1$. We call a prime $q$ of $\Lambda$ semi-discrete if it is pulled back along $\rho_{n}$ from some prime of $\Lambda /\left((1+T)^{p^{n}}-1\right)$. In other words, $q=(f)$ for some irreducible distinguished polynomial dividing $(1+T)^{p^{n}}-1$. In fact

$$
(1+T)^{p^{n}}-1=\nu_{0} \nu_{1} \ldots \nu_{n},
$$

where $\nu_{0}=T, \nu_{1}=\frac{(1+T)^{p}-1}{T}$, and in general $\nu_{k}$ is the cyclotomic polynomial of degree $p^{k}-p^{k-1}$. Thus the semi-discrete primes are precisely the $\nu_{i}$ 's, $i \geq 0$.

Similarly, a prime of $\Lambda^{\prime}$ is semi-discrete if it is pulled back from $\Lambda^{\prime} /\left((1+T)^{p^{n}}-1\right)$ for some $n$. Thus the semi-discrete primes of height one are the $\left(v_{i}, j\right), i \geq 0$, $0 \leq j \leq p-2$. Let $S_{\delta}$ denote the set of semi-discrete primes.

The semi-discrete primes play an interesting role in number-theoretic applications; for a discussion of this, see [16]. Here is another interesting example.

Proposition 10.11. Let $G$ be a discrete group, and suppose there exists a contractible finite $G$-CW-complex $Z$ with finite isotropy groups (e.g., $G$ is an $S$-arithmetic group [19]). Then $K \cdot B G$ is a finitely-generated $\Lambda$-torsion module and has support in the semi-discrete primes.

Proof. Consider first the case $G$ finite. If $P$ is a $p$-Sylow subgroup of $G$, then $\Sigma^{\infty} B G$ is a retract of $\Sigma^{\infty} B P$, since we are working with $p$-complete and hence $p$-local spectra. By Atiyah's theorem, $K^{1} B P=0$ and $K^{0} B P_{+} \cong \hat{\mathcal{R}}_{\mathbb{C}} P$ : the $p$-completed representation ring of $P$. This isomorphism is compatible with the Adams operations and hence is an isomorphism of $\Lambda^{\prime}$-modules. Furthermore, for any group $G$, the Adams operations permute the irreducible representations of $G$ (20]). Hence $K^{0} B P$ is a discrete $\Gamma^{\prime}$-module, and as a $\Lambda^{\prime}$-module is supported in the semi-discrete primes. This settles the finite case.

In the general case, we have $B G \cong E G \times_{G} Z$, since $Z$ is contractible. Let $\pi: E G \times{ }_{G} Z \longrightarrow Z / G$ denote the natural projection. Taking $\pi^{-1}$ of skeleta yields a filtration of $B G$ and hence a spectral sequence of $\Lambda^{\prime}$-modules converging to $K^{\cdot} B G$. Since the isotropy groups are finite and $Z / G$ is a finite complex, the $E_{1}$-term is a finite direct sum of modules of the form $K^{\cdot} \Sigma^{s} B H, H$ finite. Hence the $E_{1}$-term is a finitely-generated $\Lambda$-torsion module and has support in the semi-discrete primes. It follows that the same is true for the $E_{\infty}$-term, and hence for $K^{\cdot} B G$.

Proposition 10.12. The thick subcategory $\mathcal{C}_{S_{\delta}}$ is generated by each of the following two sets of objects:

a) The suspension spectra of classifying spaces $B G$, where $G$ ranges over finite cyclic p-groups.

b) The algebraic $K$-theory spectra $K \mathbb{F}_{q^{n}}$, where $q$ is a power of a prime different from $p$, and $n \geq 1$.

Proof. a) Let $\mathcal{C}$ be the thick subcategory generated by the $\Sigma^{\infty} B \mathbb{Z} / p^{n}$, and fix $n \geq 1$. Thinking of $\Gamma^{\prime}$ as the automorphism group of the $p$-power roots of unity, it is clear 
that the set of faithful irreducible representations of $\mathbb{Z} / p^{n}$ over $\mathbb{C}$ is a discrete transitive $\Gamma^{\prime}$-set with isotropy group $\Gamma_{n-1}$. Hence

$$
K^{0} B \mathbb{Z} / p^{n} \cong \bigoplus_{i=0}^{n-1} \mathbb{Z}_{p}\left[\Gamma^{\prime} / \Gamma_{i}\right] \cong\left(\bigoplus_{i=0}^{n-1} \Lambda / \omega_{i}\right) \otimes \mathbb{Z}_{p}[\Delta] .
$$

Since $\Lambda / \omega_{n} \sim\left(\bigoplus_{i=0}^{n} \Lambda / \nu_{i}\right)$ and thick subcategories are closed under retracts and pseudo-equivalences, it follows that each Moore spectrum $\mathcal{M}_{\Lambda / \nu_{n}, i}$ is in $\mathcal{C}$. Hence $\mathcal{C}=\mathcal{C}_{S_{\delta}}$.

b) Let $\gamma$ be any element of $\Gamma^{\prime}$, and consider the fibre $F_{\gamma-1}$ of $\gamma-1: K \longrightarrow K$. Then $K^{0} F_{\gamma-1} \cong \mathbb{Z}_{p}\left[\left[\Gamma^{\prime} / H_{\gamma}\right]\right]$, where $H_{\gamma}$ is the closed subgroup generated by $\gamma$. Now if $H$ is any closed subgroup of $\Gamma^{\prime}$, then either $H$ has finite index or $H \subset \Delta$. When $c(\gamma)=k \in \mathbb{Z}$ and $k>1$, the latter case cannot occur, so $H_{\gamma}$ has finite index. Furthermore, under the same assumption, $H_{\gamma^{p-1}}=\Gamma_{n}$ for some $n$, and $H_{\gamma^{(p-1) p^{m}}}=\Gamma_{n+m}$.

Thus the sequence of spectra $F_{\gamma^{j}-1}, j \geq 1$, includes the Moore spectra $\mathcal{M}_{\Lambda^{\prime} / \omega_{n}}$ for all $n$. It follows that the $F_{\gamma^{j}-1}, j \geq 1$, generate $\mathcal{C}_{S_{\delta}}$. Taking $c(\gamma)=q$ as in assertion b), we have $F_{\gamma^{j}-1}=K \mathbb{F}_{q^{j}}$, and b) follows.

\section{ACKNOWLEDGEMEnTS}

We would like to thank Ethan Devinatz, John Palmieri and Mark Hovey for helpful conversations. We would like to thank the referee for a careful reading of the paper and many suggestions for improvements.

Many of the results in this paper are from the first author's Ph.D. thesis [8].

\section{REFERENCES}

[1] Adams, J.F., Stable Homotopy Theory Lecture, Lecture Notes in Mathematics, Vol. 3, Springer, New York, 1964. MR0185597 (32:3061)

[2] Bousfield, A.K., The localization of spectra with respect to homology, Topology 18 (1979), 257-281. MR0551009 (80m:55006)

[3] Bousfield, A.K., On the homotopy theory of K-local spectra at an odd prime, Amer. J. Math. 107 (1985), 895-932. MR0796907 (87c:55010)

[4] Bousfield, A.K., A classification of K-local spectra, J. Pure Appl. Algebra 66 (1990), 120-163. MR.1075335 (92d:55003)

[5] Bousfield, A.K., and Kan, D., Homotopy limits, completions and localizations, Springer Lecture Notes in Math. Vol. 304 (2nd corrected printing), Springer-Verlag, Berlin-HeidelbergNew York, 1987. MR0365573 (51:1825)

[6] Devinatz, E., Morava modules and Brown-Comenetz duality, Amer. J. Math. 119 (1997), 741-770. MR1465068 (98i:55008)

[7] Franke, J., Uniqueness theorems for certain triangulated categories possessing an Adams spectral sequence, preprint, http://www.math.uiuc.edu/K-theory/0139/

[8] Hahn, R., University of Washington Ph.D. thesis, 2003.

[9] Hopkins, M., Global methods in homotopy theory, Proceedings of the 1985 LMS Symposium on Homotopy Theory, J.D.S. Jones and E. Rees, editors, pp. 73-96, 1987. MR0932260 (89g:55022)

[10] Hopkins, M., Mahowald, M., and Sadofsky, H., Constructions of elements in Picard groups, Topology and Representation Theory (E. Friedlander and M. Mahowald, eds.), Contemp. Math. 158 (1994), 89-126. MR1263713 (95a:55020)

[11] Hovey, M., Some spectral sequences in Morava E-theory, preprint 2004.

[12] Hovey, M., and Strickland, N., Morava K-theories and localisation, Mem. Amer. Math. Soc. 139 (1999), no. 666, x+100. MR.1601906 (99b:55017)

[13] Hovey, M., Palmieri, J., and Strickland, N., Axiomatic stable homotopy theory, Mem. Amer. Math. Soc. 128 (1997), no. 610, x+114. MR1388895 (98a:55017) 
[14] Madsen, I., Snaith, V., and Tornehave, J., Infinite loop maps in geometric topology, Math. Proc. Camb. Phil. Soc. 81 (1977), 399-430. MR0494076 (58:13007)

[15] Mitchell, S.A., On p-adic topological K-theory, in Algebraic K-Theory and Algebraic Topology, P.G. Goerss and J.F. Jardine, editors, Kluwer Academic Publishers 1993, 197-204. MR.1367298 (96h:19006)

[16] Mitchell, S.A., K(1)-local homotopy theory, Iwasawa theory and algebraic K-theory, Handbook of Algebraic K-theory, edited by E. Friedlander and D. Grayson, vol. 2, pp. 955-1010 Springer, 2005. MR2181837 (2006g:11220)

[17] Neukirch, J., Schmidt, A., and Wingberg, K., Cohomology of Number Fields, Springer, 2000. MR:1737196 (2000j:11168)

[18] Ravenel, D.C., Localization with respect to certain periodic homology theories, Amer. J. Math. 106 (1984), 351-414. MR0737778 (85k:55009)

[19] Serre, J.P., Cohomologie des groupes discrets, in Prospects in Mathematics, Annals of Math. Studies 70 (1971), 77-169. MR0385006 (52:5876)

[20] Serre, J.P, Linear Representations of Finite Groups, Springer-Verlag, 1977. MR0450380 $(56: 8675)$

[21] Washington, L., Introduction to Cyclotomic Fields, Springer-Verlag, 1982. MR 0718674 (85g:11001)

6805 Windhaven Parkway, S126, The Colony, Texas 75056

Department of Mathematics, University of Washington, P.O. Box 354350, Seattle, WASHINGTON 98195-0001 